Erschienen in: Lang, Ewald/Zifonun, Gisela (Hrsg.): Deutsch - typologisch.

Berlin, New York: de Gruyter, 1996. S. 428-470.

(Jahrbuch des Instituts für deutsche Sprache 1995)

WERNER ABRAHAM

\title{
Personalpronomina, Klitiktypologie und die Struktur des 'Mittelfeldes'
}

\author{
Wer in einer fremden Sprache schreibt, \\ der muß seine Denkungsart, wie ein Liebhaber, zu bequemen wissen. \\ Wer in seiner Muttersprache schreibt, \\ hat das Recht eines Ehmanns, \\ falls er dessen mächtig ist.
}

(Johann Georg Hamman, Versuch über eine akademische Frage)

\begin{abstract}
Personalpronomina und ihre reduzierten und klitischen Formen stehen an markanten Satzpositionen, die sich von der Position der koreferenten vollen Nomina grundsätzlich unterscheiden. Sie erscheinen allerdings in manchen Sprachen verbbezogen als Enklitika, in anderen als Proklitika. Es wird zuerst erwogen, diese enklitische bzw, proklitische Position von der grundlegenden Linearitätstypologie im greenbergschen Sinne (SVO und SOV/VSO) abhāngig zu machen. Wiewohl prinzipiell richtig zwingen klitische Pronomina im Skandinavischen sowie die Klitikstellung in Nichtdeklarativen zur Annahme, nach der ersten (rechtesten) thematischen Diskursposition im strukturellen Satzschema als Ort für dic schwachen Pronominalformen zu suchen. Dicse $\mathbf{\Lambda n}_{\mathbf{n}}$ nahme erscheint für eine Reihe von nichtverwandten Sprachen als haltbar. Im Blickpunkt stehen Sprachen aus den drei greenbergschen Haupttypen: SVO als V-mittelständigen Sprachen sowie SOV/VSO als V-randständige Sprachen. Je nachdem wie nichtdeklarative Sätze sich aus den zugrundegelegten Strukturen ableiten, erreicht das pronominale Klitikum eine enklitische oder eine postklitische Position, die sich dadurch auszeichnet, daß sie die rechteste diskursfunktionale Themaposition ist. Diese Einsicht macht die strukturelle Verschiebung der schwachen Pronomina systematisch ableit- und voraussagbar.
\end{abstract}

\section{Vorrede und Problematisierung}

Es ist auffällig, daB pronominale Klitika in jüngeren Arbeiten zur formalen Linguistik zu einem Dauerbrenner geworden sind. ${ }^{1}$ Dies hat beson-

1 So formte das vom Europarat geförderte linguistische Großforschungsprojekt Eurotyp einen eigenen Arbeitskreis zu diesem Thema; siehe Teilergebnisse in EUROTYP. PROGRAMME IN LANGUAGE TYPOLOGY. EUROPEAN SCIENCE FOUNDATION. Band 5: Clitic doubling and clitic groups sowie Band 6: Clitics: their origin, status, and position, jeweils herausgegeben von H. van Riemsdijk/L. Hellan (ohne Jahresangabe). 
dere Gründe. Wie sich zeigen wird, stehen diese phonologisch reduzierten Pronominalformen in anderen distributiven Zusammenhängen als ihre phonologisch starken Entsprechungen, so daß sich die Frage erhebt, wohin genau, d.h. an welchen strukturell vorgezeichneten Ort, die Klitika (CL) 'hinwandern' und vor allem was der Anlaß für diese Wanderung ist. Der vorliegende Aufsatz nimmt solche Fragen auf und diskutiert sie für eine Reihe verschiedener Sprachen, stets mit Blick auf die besondere Stellung des Deutschen, das solche reduzierte Pronomina mit eigenen Stellungseigenschaften ebenfalls hinreichend kennt. Folgende Feststellungen sind bereits erhärtet bzw. ergeben sich jeweils aus vorigem:

(i) Konservative Dialekte des Deutschen zeigen ausschlieBlich phonologisch reduzierte Formen bzw. klitische Entsprechungen (CL) der personalen Vollpronomina (Pron). Im besonderen gibt es keine Möglichkeit, Personalpronomina betont zu verwenden. Ist dies die ur- oder prototypische Funktion von CL? Worin ist diese Erscheinung begründet? Zur Feststellung, da $\beta$ pronominale Klitika nicht in der Basisposition der koreferentiellen Objekte innerhalb von VP stehenbleiben können, vergleiche man die folgenden Beispiele aus dem modernen umgangssprachlichen Deutsch.

(1) a daß-s (= es, das Kind) dem Vater gefolgt hat

Träger = COMP (Konjunktion)

b Was hat-s dem Vater gegeben?

Träger $=$ finites $V$

c Hat-n der Vater gesehen?

Träger $=$ finites $\mathrm{V}$

d ${ }^{*}$ da $B$ dem Vater-s gefolgt hat

Träger ungleich COMP, nämlich Dativobjekt

e *Was hat dem Vater-s gegeben?

Träger ungleich finitem V, nämlich Dativobjekt

f *Hat dem Vater-s gefolgt?

Träger ungleich finitem $V$ (Dativ)

g *-n hat der Vater gesehen? (Satzspitze)

h S-ist einmal ein kleines Männchen gewesen. (Satzspitze) nur phonolog. Bedingg. (geringe Sonoritāt) gegeben

i *R-hat einmal ein kleines Männchen gesehen. (Montafonerisch) schnellsprachl. R- bzw. 'r für Er:

phonolog. Bedingg. alleine reicht nicht

Klitische Positionen (Positionen von 'pronominalen Klitika' = CL) sind eben nicht identisch mit den koreferenten NP-Positionen. Sie hängen 
zum einen, so wollen wir vorwegnehmen, von bestimmten 'Trägerkategorien' ab - das ist die phonologische Bedingung. Vgl. auch (1g), wo ja ein derartiger phonologischer Träger nicht gegeben ist im Vergleich zu (1c). Solche phonologischen Bedingungen alleine können aber die CLErscheinung nicht erklären, wie $(1 \mathrm{~h}, \mathrm{i})$ zeigen. Vielmehr hängen CLPositionen und ihre Verteilungscharakteristika weiter ebenso notwendig, aber darüber hinaus erst hinreichend, von bestimmten syntaktisch bestimmten Stellungen im Satz ab; vgl. wieder (1a-c) und (1d-f). Es wird zu zeigen sein, daß die syntaktische Bedingung durch alle hier untersuchten (idg.) Sprachen hin streng eingehalten wird, aber von typologischen Determinanten der jeweiligen zugrundeliegenden Satzstruktur abhängig ist.

(ii) Entgegen Wackernagels (1892) Generalisierung gibt es klitische Pronomina in Satzerstposition (vgl. Taylor 1993 zum Altgriechischen oder Eythórsson (1994) zu einer Reihe frühgermanischer Dialekte). Steht dies im Widerspruch zu (i)?

(iii) Die Frage danach, welcher grammatischen Kategorie der Klitikträger angehören muß, ist auf den ersten Blick verwirrend: in SOV-Sprachen müssen die Träger entweder finite (Hilfs-) Verben oder unterordnende Konjunktionen sein. Andere Kategorien sind ausgeschlossen. Vgl. (1ai). Jedoch: was sind die Gemeinsamkeiten zwischen finiten Verben und einbettenden Konjunktionen derart, daß sie diese Eigenschaft teilen können? Bei SVO-Sprachen sieht dies anders aus, da von Trägern im engeren Sinne bei den proklitischen Stellungen gar nicht gesprochen werden kann (Italienisch, Französisch) - bei den enklitischen wie im Rumänischen oder Italienischen hingegen wohl. Die Frage ist auch berechtigt, wieso denn CL im Romanischen (und allgemein in SVO-Sprachen) nicht an COMP wie im Deutschen herantreten. Spielt COMP im Romanischen keine so ausgezeichnete Rolle wie im Deutschen (genauer: beim Typus 'SOV+V-Zweit', den das Deutsche, Niederländische und Westfriesische ja mit der Scheidung zwischen abhängigem (SOV) und unabhängigem (V-Zweit) Satz darstellen)?

(iv) Pronominale Klitika (CL) sind unabhängig davon, ob sie spezifische, von den vollpronominalen Formen verschiedene phonologische Formen aufweisen, syntaktisch-positionell ausgezeichnet. Dies gilt für CL in beiden Sprachtypen: SOV ebenso wie SVO. Die syntaktischen Positionen von starken pronominalen Formen (Pron) und ihren klitischen Entsprechungen (CL) sind nicht identisch. 
(v) Unabhängig von der typologischen, greenbergschen Unterscheidung stehen CL ausschlieBlich links von ihren vollpronominalen Entsprechungen.

(vi) Wo Kasusunterscheidungen eine Rolle spielen wie im Deutschen, stehen Subjekt-CL immer links von Objekt-CL. Bei der DativAkkusativabfolge dreht sich die Abfolge um: NP-3+NP-4 $\neq$ CL-4+CL-3. Beide Erscheinungen erfordern eigene syntaktische Erklärungen.

(vii) Typologische Zugehörigkeiten alleine erlauben keine Voraussage darüber, ob CL auftreten. Das Serbokroatische und das Bulgarische 2.B. erlauben sich den Luxus von CL-Gruppen (CL clusters) und CL-PronKopien, während das Russische überhaupt keine CL besitzt. Man vgl. auch den Unterschied zwischen Altgriechisch und Lateinisch: letzteres hat uns keine CL überliefert.

(viii) ALLGEMEINE THESE ZUR CL-TYPOLOGIE: Es wird hier zuerst die empirisch und kausal weiter plausibel zu machende Position verfolgt, daß die Stellung der klitischen Pronomina (CL) - sofern in einer Sprache überhaupt pronominale CL auftreten - typologisch bestimmt ist: V-interne Sprachen (SVO, OVS (?)) zeigen Proklitika, V-periphere Sprachen (SOV, VSO, VOS (?)) dagegen Enklitika.

(ix) Es wird sich allerdings zeigen, daß die allgemeine stellungstypologische These in (viii) nach bestimmten Details zu präzisieren und zu modifizieren ist und zwar folgendermaßen:

SPEZIELLE CL-THESE: Pronominale CL sind hochthematisch ('thematisch' als Diskurskategorie, in Opposition zu 'rhematisch'). Sie stehen demgemäß in jener thematischen Position, die den geringsten Abstand zu den koreferenten nominalen Entsprechungen aufweist. Im Szenario eines Verschiebungsmechanismus der CL aus der VP heraus nach links heiBt dies, daB CL in der erstmöglichen thematischen Position stehen bleibt. Wir haben diese diskursstrukturelle Satzperiode, in die das thematische pronominale CL zu stehen kommt, FP ('Funktionalphrase') genannt und es weiterer empirischer Arbeit vorbehalten, diese vorerst diskursfunktionale Kategorie FP jeder Sprache extra auf die universalgrammatisch relevanten strukturellen Satzperioden abzubilden.

Mit dieser Präzisierung ist auch die Begründung gegeben (vgl. auch (x) unten): Klitische Pronomina rücken aus der Basisposition für die entsprechenden koreferentiellen NPs nach links heraus und zwar so weit nach links - entweder in das 'Mittelfeld' oder noch weiter über das finite Prädikat hinaus -, bis sie die erste mögliche Position im satzstrukturellen Diskursthemabereich, also den Bereich links von VP erreicht haben. Es ist unmittelbar deutlich, daß unter solchen Arbeitsprämissen ein 
nichttriviales, detailliert formalisiertes sowie eben dadurch einer empirischen Detailüberprüfung zugängliches Konzept einer satzsyntaktischen Struktur zugrundezulegen ist. Ganz konkret: es muß unmittelbar und einleuchtend verständlich werden, unter welchen Bedingungen die typologisch gröbere These in (viii) zugunsten von (ix) aufzugeben ist und wieso dann - d.h. unter welchen sehr spezifischen Zusatzkriterien, die sich aus dem Zusammenspiel zwischen diskursfunktionalen Kategorien wie Thema und Rhema und einer empirisch wohlbegründeten syntaktischen Untergliederung des Mittelfeldbegriffs ergeben - die These in (viii) doch noch sinnvoll bleibt.

(x) Wenn (viii) und (ix) empirisch haltbar sind, dann erhebt sich die Frage, wie sich diese deutlich unterschiedliche Verteilung zwischen pronominalen CL und deren koreferentiellen NPs begründet. Wie in (ix) angedeutet werden Argumente der Thema-Rhema-Verteilung ins Treffen geführt: je schwächer Pronomina, desto thematischer ist ihre Diskursleistung. Dies wirkt sich durch Verschiebung nach links in den satzstrukturellen Themabereich aus und führt $\mathrm{zu}$ folgendem Arbeitsprogramm:

(x.1) Für den Landeplatz der diskursfunktional motivierten CL-Linksverschiebung nimm an, daB CL nicht weiter als zur grammatischen Satzkategorie FP vorrückt.

(x.2) FP, Maximalprojektion mit kanonischer Projektionsgliederung (vornehmlich einem [Spez,P] mit $\mathrm{Spez}^{\circ}$ sowie einem Kopf, $\mathrm{F}^{\circ}$ ), ist der rechte Randknoten in einer universalgrammatischen Satzstruktur, dessen Kernbereich in der diskursthematischen Satzperiode liegt. Vgl. (34) unten.

(x.3) Weder ist FP hinsichtlich der kategorialen Alternative 'Argument/Adjunktion' festgelegt, noch ist CL von vornherein als XP, also als Maximalprojektion bestimmt. Beide Optionen sind nach einzelsprachlichen Distributionsdispositionen festzulegen. ${ }^{2}$

So weit zum Programm des Aufsatzes. Nun zur Ausführung. Zuerst diskutiere ich in Abschnitt 2 den kategorialen Status von Pronomina und ihren klitischen Formen, CL. In Abschnitt 3 stelle ich verschiedene Sprachen der drei Typen SVO, SOV und VSO mit ihren CL-Vertretungen vor. Abschnitt 4 gibt einen Überblick über distributionelle Gemeinsamkeiten und Unterschiede zwischen SVO- bzw. V-randständigen Sprachen. In 5 werden die diskursfunktionalen Satzperioden Thema und Rhema auf die universalgrammatische Satzstruktur abgebildet und der genaue syntak-

2 Fürs Deutsche nehmen Abraham/Wiegel (1993) an, daß CL kategorienselektiv an Köpfe rechtsadjungieren (also enklitisch stehen). Aufgrund des Strukturerhaltungsprinzips folgt damit, daß CL als Nullprojektionen, also $\mathrm{N}^{\circ}$ bzw. $\mathrm{D}^{\circ}$ zu sehen sind. 
tische Ort von CL, schwachen Pronomina sowie starken Pronomina und Vollnomina festgelegt. In Abschnitt 6 gehe ich kurz auf eine rätselhafte Inversionserscheinung ein. In Abschnitt 7 wird die spezielle typologische CL-These anhand dreier Konstruktionstypen (Infinitiven, Imperativen und Gerundien) diskutiert. In Abschnitt 8 werden die unserer allgemeinen typologischen These scheinbar widersprechenden skandinavischen Sprachen auf ihre CL-Eigenschaften hin überprüft, was direkt zur speziellen CL-These in (ix) führt. Abschnitt 9 schafft einen abschließenden generellen Überblick.

\section{Syntaktische CL-Charakteristika bei SOV}

$2.1 \quad$ NP- (= Konstituenten-) Status, XP, oder Einzelwortstatus, $\mathrm{X}^{\circ}$ ?

Es ist für die Analyse nicht unerheblich, was der Projektionsstatus von starken, schwachen und CL-Pronomina ist. Man vgl. dazu die folgenden Selektionsbeschränkungen.

(2) *der er-außer st. Pronomina modifiziert durch einen Relativsatz: er, der-n gesehen hat

(3) *schöner er

(4) *er Mann

Daraus geht eindeutig hervor, daB der Wortartstatus der einer DP, nicht bloß einer NP ist. D.h. der er bzw. er Mann gehen deshalb nicht, weil er bereits den bestimmten Artikel (D) ebenso wie den N-Kopf soz. inkorporiert. Wie ist der janusköpfige Projektionsstatus aufzulösen? Es ist ja nicht vorstellbar, daß das Pronomen sowohl D wie auch $\mathrm{N}$-Kopf ist. Nun sind wir bei CL relativ sicher: das starke Pronomen ist von Maximalprojektionsstatus, also eine DP. ${ }^{3}$ Da sich CL andererseits an einen Kopf mit dem Status von $\mathrm{X}^{\circ}$ affigiert (COMP, V; man denke an die Konjunktionsflexion im Bairisch-Österreichischen), muß nach einem sehr allgemeinen Strukturerhaltungsprinzip CL von derselben Projektionsklasse sein: $\mathrm{X}^{\circ}$. Da es sowohl D-Referenz als auch Kopfstatus haben muß, ist es ein

3 nach herkōmmlicher Annahme mit folgender Struktur:<smiles>[Pb]</smiles>

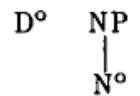


D-Kopf, also $\mathrm{D}^{\circ}{ }^{4}$ Dies trägt dem Umstand Rechnung, daß bei Enklitisierung wie im Deutschen Rechtsadjunktion vorliegt. Wenn jedoch, was sich nicht so leicht nachweisen läBt, CL in einem eigenen Funktionsknoten innerhalb von IP steht, dann ergibt sich, sofern wir Verschiebung aus VP annehmen, ein Lizensierungsproblem: eine DP, verschoben aus VP heraus in die thematische Strukturperiode, kann nur in eine Kopfposition rücken, wenn auch die Spezifikatorstelle, die DP vertretende Stelle, besetzt ist. Daraus ergibt sich ein Lizensierungsproblem: wir nehmen - im üblichen formalen Jargon - an, daB die Spez-Stelle mit einem pro besetzt ist, das seinerseits (über Spez-Kopf-Rektion) das Kopf-CL lizensiert.

Diese strukturellen Vorgaben haben auch zwingende diskursfunktionale Foigen. Erstens sind nichtfokussierte bzw. nichtfokussierbare Elemente links von VP vom Thema-Hintergrund-Status. Vgl. zu Genauerem (34) unten. Zweitens ist der Projektionsstatus (vgl. die Diskussion zu (2)-(4)) ein kritischer Parameter: Maximalprojektionen (XP, somit in Sonderheit Artikelkonstituenten, DP bzw. [Spez, CP], die Satz-TOP-Position) sind definit und deshalb im allgemeinen referentiell spezifisch, eben auf keinen Fall referentiell unspezifisch und indefinit. Die Minimalprojektion $\mathrm{X}^{\circ}$, diejenige, in der wir Klitika identifiziert haben, muß aufgrund der prinzipiellen Spez-Kopf-Beziehung ebenfalls definit und referentiell spezifisch sein. Den Unterschied zwischen diesen beiden Positionen lokalisiere ich in der Fokussierbarkeit: DP (in Spez-TopP) kann immer Satzakzentträger sein, die dazugehörige $\mathrm{X}^{\circ}$-Position dagegen nie. Sie lassen sich aus diesem Grunde mit den beiden ersten diskursfunktionalen Perioden in (34) identifizieren: sie haben gemeinsam diskursfunktionale Themageltung, unterscheiden sich aber mittels der Kriterien 'Vordergrund' gegen 'Hintergrund'. Was den Spezifizitätscharakter von CL (und des NP-Subjekts) im besonderen ausmacht, sind die Merkmale der Person und des Numerus relativ zur Sprecherperson und somit auch der Personund Numeruskongruenz. Während starke Pronomina (die ja auch reinen DP-Charakter haben) selbst Identifikationen nach Person und Numerus erlauben, sind CL im D-Status (haben also Kopfcharakter bei DStatus, den sie mit starken Pronomina teilen), sind also wie alle Artikelwörter nicht nach Person identifiziert. Diese Identifikation muß durch einen Lizensierungsmechanismus erwirkt werden, der referentielle, also pragmatische Indizierung nach Personenreferenz erlaubt. Ich nehme an, $\mathrm{da} \beta$ eine solche Indizierung dem thematischen Vorfeld vorbehalten bleibt.

4 Cardinaletti (1994, S. 198) faßt dies in folgender 'flachen' Struktur: D $^{\circ}$ 
Somit mu $B$ jedes pronominale $\mathrm{CL}$ in diesen diskursfunktionalen Bereich vorrücken. ${ }^{5}$ Und mit Kopfstatus als $\mathrm{D}$ (et(erminer)) ist ihm eine Kopfposition in TopP vorbehalten: CL kann nur so weit 'aufsteigen', bis es eine derartige Stelle erreicht hat, in der Thematizität in Ableitung von der Spezifizität bezügl. Person gesichert ist. Starke Pronomina haben diese Spezifizität in situ, also innerhalb von VP so wie NPs.

$\mathrm{DaB}$ CL nicht weiter steigen als bis zum erstmöglichen thematischen Kopfknoten, zeigen Verbkomplexe im Französischen sowie COMPHäufungen im Niederländischen und Deutschen.

(5) a Nous (*te) voulons te voir $\left({ }^{*}\right.$ te)

Frz. (nach Uriagereka 1995, S. 106: sein Beispiel (26))

b $\quad \ldots$ weil $<{ }^{\prime *} n>$ daB $<$ 'n $>$ der Teufel geholt hat bair.-ōsterr. Dialekte

c $\quad$... voor $<$ '* $\mathrm{t}>$ dat $<$ ' $\mathrm{t}>$ iemand zag ndl. Standard vor daB es-CL jemand sah

Damit sind wichtige Korrelationen zwischen diskursfunktionalen Satzperioden und syntaktisch strukturellen Kategorien erkannt. Es zeigt sich einmal mehr, daß sich die strukturellen Abschnitte der Diskursfunktionen Thema und Rhema in Abhängigkeit von einer entsprechend leistungsfähigen Satzsyntax aus begründen lassen.

\section{Pronomina und ihre klitischen Formen in anderen} Sprachen

3.1 SVO-Sprachen. Romanische Beispiele (SVO):

FRANZÖSISCH (Kayne 1975):

(6) a *Marie ne connait que les ...*L Pron-Form

M. NEG kennt außer sie

"Marie kennt nur sie"

b Marie les connait ... CL

CL in linksverschobener Stellung

5 Uriagereka (1995) nimmt, um eben dies zu erreichen, einen eigenen funktionalen Knoten, FP, über dem Flexionsknoten, IP (oder dessen Satelliten AgrS bzw. AgrO) an, der diese Attraktion auf CL ausübt. Es bleibt aber unklar, warum dieser funktionale Knoten gerade dort sitzt, wo er von Uriagereka hingesetzt wird - wenngleich er denselben Horizont anstrebt, wenn er sagt ,... F, the syntactic element interfacing with pragmatic indexicality." (Uriagereka 1995, S. 93) 
c Marie ne connait qu'eux $\ldots{ }^{*} \mathrm{CL}$, Pron-Form Pron bei rechtsläufiger Rektion

M. NEG kennt außer euch

$d^{*}$ Marie eux connait

... * Pron-Form in CL-Position

Natürlich ist $j e$ bereits klitisch, da es nur präverbal stehen kann. Postverbal, in Fokusposition, muB es ja durch eine Suppletivform vertreten $\operatorname{sein}(m o i)$.

ITALIENISCH (Cardinaletti 1994):

(7) a *Maria conosce ci

$\ldots{ }^{*} \mathrm{CL}$, Pron-Form

b Maria ci cognosce

$\mathrm{CL}$ in linksverschobener Stellung

c Maria cognosce noi

Pron bei rechtsläufiger Rektion

$\mathrm{d}^{*}$ Maria noi cognosce

...*Pron-Form in CL-Position

RUMÄNISCH (Dobrovie-Sorin 1994, S. 70):

(8) a L-am rugat

ihn=habe(-ich) gebeten

b *am il/o rugat

habe(-ich) ihn/sie gebeten

c baiatul pe care l-am vazut

der Junge P-den ihn=habe(-ich) gesehen

$\mathrm{Da} B$ allerdings das klitisch-pronominale Femininum als Objekt enklitsch und nicht proklitisch wie alle anderen schwachen Pronomina erscheint, läßt den Schluß zu, daß bei Klitisierung phonologische Prozesse von syntaktischen zu trennen sind. Dies ist angesichts des Sonderverhaltens von deutsch es bzw. 's sowie ndl. ' $t$ nicht überraschend. Siehe $(9 \mathrm{a}-\mathrm{c})$ - es handelt sich hierbei eben nicht um syntaktische CL, sondern um Schnellsprechformen ohne syntaktische Systematisierbarkeit.

(9) a Am rugat-o ... rumän. CL habe(-ich) gebeten=sie

b 's ist einmal/'s hat einst/?? s' atmet noch in TOP-Position: nur für expletives es, nie für es(N), sie(F)

$c$ ' $t$ is nu eenmaal zo ndl. Expletivum, nie statt het(N)

Ebenso wie im Deutschen erscheint auch im Rumänischen die Konjunktion oder die Negationspartikel als phonologischer CL-Träger; man be- 
achte, daß dieser Umstand nicht an der syntaktischen Prokliseeigenschaft rüttelt:

(10) Nu stie ca-l/c-o/ca-i asteapta mama

Nicht wei $B(-e r), d a B=i h n / d a \beta=s i e / d a \beta=s i e P L$ erwartet Mutter

Slawische Beispiele (Bulgarisch, Makedonisch, Serbokroatisch und Tschechisch, alle SVO; nach Dimitrova-Vulchanova 1993):

\section{BULGARISCH}

(11) a Ne si li mu ja dal knigata?

CL-Häufung nach der Generalisierung li+AUX-CL+PRON-CL

NEG hast Q-PARTIKEL ihm es gegeben Buch-das?

b Ste mu go pratja

kanonische Kasusabfolge: DAT + AKK

werden ihm es schicken

c Knigata dali mu ja dadoxa?

Buch-das ob ihm es gaben?

"Haben sie ihm dieses Buch wirklich gegeben?"

d Toj kaza, ce knigata sum mu ja bil dal

er sagte daß Buch-das habe(ich) ihm es gehabt gegeben

e Toj kaza, ce na tebe knigata sum ja bil dal

er sagte daß DIR Buch-das habe(ich) es gehabt gegeben

Wie (11d, e) zeigen, steht das nichtklitische Pronomen - im obigen Fall das emphatische na tebe "dir" - in einer anderen syntaktischen Position als CL.

\section{MAKEDONISCH}

(12) a Go vidov nego

CL-Dopplung (umggsprachl.)

ihn sah (ich) ihn

b Zima ja imase pritisnato Struga

Winter es hatte gepackt (die Stadt) Struga

c Jas sum mu gi zel parite ich habe ihm sie weggenommen Geld-das

d Ja vidov Marija/zenata sie sah(ich) Maria/Frau-die

e Daj mu go gib ihm es

\section{SERBOKROATISCH}

(13) a Ja mu ga zelim dati

ich ihm es möchte geben 
b $\quad$ Taj < pesnik>mi je < pesnik>napiso knjigu dieser (Dichter) mir hat (Dichter) geschrieben Buch-ein

c Zelim da mu ga dam möchte(ich) zu ihm es gebe(ich) = 'ich möchte zum ihm es geb(e)' vgl. $z u m=8=m$ geben oder $z u m=8=n=$ unschädlich machen

\section{TSCHECHISCH}

(14) a Ma te Jan rad? hat dich Jan gerne?

b Nevidel jsem te cely den NEG=gesehen habe dich den ganzen Tag "Ich habe dich den ganzen Tag nicht gesehen"

c Nemel jsi ho urazet $\mathrm{NEG}=$ solltest haben ihn beleidigt

Zu beachten ist, daß Proklitizität im Slawischen, wie oben dargestellt, nicht mit Blick auf das finite Hilfsverb, sondern auf das (oft infinite) Vollverb gilt. Mit andern Worten: wo im Romanischen oberflächlich $\mathrm{CL}=$ $A U X+V$ gilt, setzt das Slawische, jetzt einmal von anderen Bedingungen ganz abgesehen, $A U X=\boldsymbol{C L}+V$. Dies soll als erste wesentliche Schärfung des am greenbergschen Typologieinventar orientierten Begriffsapparats gesehen werden: Pro- bzw. Enklitizität sind jedenfalls - und sehr wahrscheinlich nicht ausschließlich - mit Blick auf die syntaktische Periode zwischen finiten und infiniten Komponenten eines komplexen Prädikats zu bestimmen. Wir kommen noch ausführlich dazu.

Zum klitischen Status des Reflexivpronomens und seiner spezifischen Verwendung als Diatheseanzeiger, also in der Funktion eines Verbgenusmorphems im Deutschen und einer Reihe anderer Sprachen vgl. Abraham (1995a). Der klitische Status des 'diathetischen' Reflexivs mag nicht gleich ersichtlich sein. Sofern nicht durch das Intensivierpronomen verstärkt (und nur dann fokussierbar: sich selbst) sind sich und seine Vertreter der 1. und 2. Person stellungsbeschränkt und zwar im Sinne der Klitikrestriktionen auf die Position nach V bzw. COMP. Klitikstatus sollte sich aber auch im Romanischen durch Proklisis erweisen; dies bestätigt sich, etwa am Rumänischen (Dobrovie-Sorin 1994, S. 169ff.; vgl. auch Grosu 1994, Kap. 7 sowie Farkas/Zec 1995):

\section{RUMÄNISCH}

(15) a Nu se poate dormi cu atta zgomot nicht se kann schlafen bei all dem Lärm „Man kann bei all dem Lärm nicht schlafen" 
b S-a spus câ Ion e nebun

se=hat gesagt daß Hans ist verrückt

"Man sagt..."

(15b) zeigt die orthographisch signalisierte Präinkorporation an den finiten Prädikatteil. In der Bedeutung ähnelt dies natürlich dem siimpersonale der italienischen Grammatik bzw. dem on dit des frz. Reflexivs (Abraham 1995a, S. 34-40; zu viel Genauerem dazu vgl. DobrovieSorin 1994, S. 175ff.). Wie sehr dieses deagentivierende Reflexiv bereits aus dem Pronominalparadigma herausgetreten ist und Verbeigenschaften wie Diathese anzeigt, läßt sich lebendig auch am Deutschen zeigen.

(16) a Es läßt sich Armen Almosen besser geben als Reichen.

b Es lassen sich Almosen Armen besser geben als Reichen. ${ }^{6}$

Die Objektvalenz ist in (16) voll abgesättigt, keinesfalls durch das Reflexiv mitübernommen. Wenn aber sich kasuslos ist, kann es nicht mehr Pronomen bzw. in einer nominalen Satzgliedfunktion sein (Kasusfilter). Zudem zeigt sich ja keine koreferentielle Bindung an ein Antezedens insofern tritt dieses 'denaturierte' Reflexiv auch aus anderen syntaktischsemantischen Zusammenhängen, in denen es sich sonst universalgrammatisch begründet. Die diathetische Funktion dieser Reflexivverwendung hängt offenbar damit zusammen, daß koreferentielle Reflexivität die Passivableitung blockiert (so Fanselow 1991, der diese Bedingung jedoch nicht weiter begründet): aus $X$ wäscht sich ist kein Passiv, etwa ${ }^{*} E r / s i c h$ wird durch $X$ gewaschen abzuleiten. Dies ist keineswegs unmittelbar einsichtig, da ja die sonstige (und einzige) Passivbedingung im Deutschen, nämlich Agentivität des Prädikats wäscht vorliegt. ${ }^{7}$

6 Die beiden bedeutungsmäßig identischen Sätze haben unterschiedliche syntaktische Strukturen: (a) ohne Prädikatkongruenz mit dem referentiellen Subjekt hat ein strukturell höheres Expletivum es, also in CP, als (b), wo das subjektkongruierende Prädikat es tiefer, also in $\mathrm{AgrS}$, vorzusehen ist. $\mathrm{Zu}$ solchen Argumentationen vgl. Abraham (1992b).

7 Wenn wir davon ausgehen, daß Prinzip A der Bindungstheorie zur tieferen Begründung des Reflexivpronomens irgendwo erfüllt sein muß, dann muß dies in der Komponente der Logischen Form, LF, gegeben sein, jener Komponente, wo alle lexikalischen und morphologischen Elemente ihre volle funktionale und logisch-semantische Interpretation erhalten. Dies wäre so vorstellbar, daß das direkte Objekt an die Stelle des in Mittelkonstruktionen wie (16) notwendigen expletiven es rückt und die Funktion des für die Bindungsbeziehung unverzichtbaren Antezedens übernimmt. Allerdings trāgt dieses Szenario im Deutschen deswegen nicht, weil es ja auch intransitive Mittelkonstruktionen gibt, wo also kein direktes Objekt für die Rekonstruktion des Antezedens in LF herhalten kann. Es bleibt somit nur die Bindung an die agentische Thetarolle, die ja in LF sichtbar wird. Damit wird aber 


\section{$3.2 \quad$ Typologische Erweiterungen}

\subsubsection{Modernes Arabisch und Hebräisch}

Das Arabische ist vom Typ SVO mit distributionellen VSO-Spuren; vgl. Fassi-Fehri (1993). Die Beispiele hier stammen aus Shlonsky (1994) ('=' notiert die CL-Bindung an den lexematischen Träger). Das moderne Hebräisch, wiewohl heute SVO, hat noch Spuren des alten VSO-Typs bewahrt.
a $[$ kaan bixayyt $]=$ ha
'Er nähte es gerade' (er) war nähend-3FS modernes palästinensisches Arabisch:[V-fin] $=\mathrm{CL}$
b asaan-ha bitxayyt l-fistyaan... 'weil sie näht das Kleid' $\mathrm{COMP}=$ Subj-CL
c tmunot-eha tluyot al ha-kir Modernes Hebräisch Bild-3FS hängt an der-Wand Subj-NP=Poss-CL 'ihr Bild hängt an der Wand'
d xasavnu al-eha (wir) dachten an-3FS
Modernes Hebräisch 'Wir dachten an sie'

\begin{tabular}{|c|c|c|}
\hline a VERB + OBJECT & $\begin{array}{l}\text { fhim l-malme } \\
\text { (Ich) verstand den Lehrer }\end{array}$ & $\begin{array}{l}\text { fhimt-ha } \\
\text { (Ich) verstehe sie }\end{array}$ \\
\hline b N + POSSESSOR & beet l-malme & beet-ha \\
\hline & $\begin{array}{l}\text { Haus der-Lehrer } \\
\text { 'des Lehrers Haus' }\end{array}$ & Haus-ihr \\
\hline c $\mathrm{P}+$ OBJECT & $\begin{array}{l}\text { min l-malme } \\
\text { von dem-Lehrer }\end{array}$ & $\begin{array}{l}\text { min-ha } \\
\text { von-ihr }\end{array}$ \\
\hline $\mathrm{d}$ CONJ + SUBJECT & innu l-malme & in-ha \\
\hline e QUANTIFIER+NP & $\begin{array}{l}\text { das der-Lehrer } \\
\text { kull l-malmaat } \\
\text { all die-Lehrer }\end{array}$ & $\begin{array}{l}\text { das-sie } \\
\text { kull-hin } \\
\text { alle-sie }\end{array}$ \\
\hline
\end{tabular}

(19) a il mudarris fahhim-u li l-bint Kairoter Arabisch der-Lehrer CAUS-verstehen-es dem-Mädchen

Objektpronominalisierung

umso mehr die verbmorphologische diathetische Leistung des klitischen Reflexivs sichtbar und begründet (so schon Abraham 1988a; ebenso Abraham 1994, Kap. 12). 
b il mudarris fahhim-ha l-dars

der-Lehrer CAUS-verstehen-sie die Stunde

nur ein einziges CL an V, das andere als PP innerhalb VP

c il mudarris fahhim-u laa-ha

der-Lehrer CAUS-verstehen-es ihr $\quad \mathrm{V}+\mathrm{DO}-\mathrm{P}+\mathrm{IO}$

d *il mudarris fahhim-ha-u/u-ha

der-Lehrer CAUS-verstehen-ihr-es/es-ihr

Kein Clustering: ${ }^{*} \mathrm{P}+\mathrm{IO}+\mathrm{DO}$

\subsubsection{Walisisch:}

Das Walisische ist wie alle keltischen Sprachen mit Ausnahme des Bretonischen, heute und in seinen historischen Vorstadien (Lewis/Pedersen 1937, S. 267ff.; Schafer 1995; Ternes 1970; Evans 1976: 179ff.; Thurneysen 1966, S. 327) streng und unkontrovers VSO, mit präverbalen Verbpartikeln, die die Satzart (affirmativ bzw. interrogativ) sowie Satznegation (vgl. hierzu besonders Ternes 1970 und Schafer 1995 fürs Bretonische) festlegen. Personalpronomina - und nur diese, nicht andere wie Possessivpronomina, die als Nominalpräfixe erscheinen, oder die erwähnten Satzartpartikel -, die klitisch ('abhängig', als grammatisch gebundenes Morphem) sowie frei vorkommen, standen und stehen als Suffixe (also 'enklitisch') oder infigiert (Lewis/Pedersen 1937, S. 193ff.; Mittelwalisisch: Evans 1976, S. 49f.). ${ }^{8}$ Subjektpronomina haben oft keine klitischen Vertreter, sondern werden nur durch die Kongruenzendung am Verb ausgedrückt (so auch Lewis/Pedersen 1937, S. 194; somit eingeschränkte 'pro-drop'Erscheinung). Es werden (nach Roberts/Shlonsky 1994) heute 16 unterschiedliche und weitgehend heterogene CL-Klassen verzeichnet, durchaus mit unterschiedlichen Stellungen zum Trägerlexem. Siehe zur Heterogenität (20).

Possessivpronomina unterschiedlicher syntaktischer Herkunft, freie Klitika der Klasse 1, erscheinen wie oben angedeutet unveränderlich proklitisch bzw. (in der Terminologie von Ternes (1970), allerdings auf das

8 Es ist nochmals zu betonen, daß es hier nur um die besondere Stellung der Personalpronomina und deren 'Herkunft' aus den koreferentiellen NPStellungen geht, nicht jedoch um eine unifizierende Erklärung der Stellung aller klitischen Morpheme, d.h. eine Beschreibung und Erklärung über alle unterschiedlichen Kategorien und Herkünfte hinweg. Damit verfällt im besonderen die Kritik von E. Ternes, der auf den proklitischen bzw. präfigalen Charakter der bretonischen Possessivpronomina und Verbpartikel verwies - welche Erscheinung ja gegen meine hypothetische typologische Verallgemeinerung zu sprechen schien. 
Bretonische beschränkt, 'präfigiert') mit Trägerkategorien wie $N, V$ und CONJ:
a ei wraig ${ }^{9}$ seine Frau
b Mae Megan wedi ei weld 'M. hat ihn gesehen' ist $M$. nach seinem Sehen
c Dywedodd y bachgen ein bod wedi cyrraedd sagte der Junge unser Sein-Nach-Ankommen
'Der Junge sagte, daß wir angekommen sind'

Diese Präfixklasse von CL mit den spezifischen Selektionsbeschränkungen unterscheidet sich von den meisten der anderen 15 CL-Klassen grundlegend. (20) ähnelt dem traditionellen nominalen Gerundium. Man beachte, daB hierbei nicht wie im Satzverband Thema- und Rhemafunktionen zu unterscheiden sind, die als treibende funktionale Kraft für die CL-Positionen verantwortlich gemacht werden. Die Erscheinungen dieser Klasse bleiben weiter unberücksichtigt. Was uns hingegen brennend interessiert, ist, wie sich CL im freien Satzverband verhalten. Die Personalpronomina, Klasse 2, nun erscheinen in solchen syntaktischen Umgebungen und zwar als Kongruenzpronomina enklitisch als Suffixe oder als unfreie Suffixmorpheme, beim nichtzusammengesetzten Verb bzw. bei der Präposition (Lewis/Pedersen 1937, S. 282; Roberts/Shlonsky 1994, S. 3). ${ }^{10}$

(21) Mae Emrys yn ei roi iddo

ist Emrys in seinem Geben=ihm Enklise; nie Traubenbildung

"Emrys gibt es ihm"

(22) a Roeddent hwy wedi clywed [PP yddint ennil y gadair]

waren=sie nach Hören für=sie Erringen den Lehrstuhl

P-Kongruenz

„Sie hatten gerade erfahren, daß sie den Lehrstuhl errungen hatten"

b Mae Emrys yn ei roi iddo

ist Emrys in seinem Geben es ihm keine Traubenbildung!

${ }^{9}$ Ich danke E. Ternes (Hamburg) für insistente Aufklärung.

10 Lewis/Pedersen (1937, S. 282) erwāgen - das ist gegen den Hintergrund der Ausgangsthese, wonach SOV und VSO eine gemeinsame Strategie gegenüber schwachen Pronomina und $\mathrm{CL}$ entwickeln, nicht uninteressant -, $\mathrm{daB}$ aus dem Idg. ererbte Verbpersonalendungen den keltischen dermaßen ähnelten, daß sie in das neue System inkorporiert werden konnten. 
c Mae Megan wedi ei weld o/*Emrys ist Megan nach seinem Sehenihn/*Emrys Beschränkung der Postposition zu V auf CL

d Dywedodd bachgen ei bod hi/*Megan wedi cyrraedd sagte der Junge ihr Sein sie/*Megan nach Ankommen

In der folgenden Übersicht sind die wesentlichen Gemeinsamkeiten und Unterschiede der CL und sw. Pronomina im modernen Arabischen und Walisischen einander gegenübergestellt.

(23) CL-Status und distributionelle Eigenschaften (nach Roberts/ Shlonsky 1994, S. 4).

\begin{tabular}{|l|l|}
\hline WALISISCH & ARABISCH \\
\hline kein Clustering & $\begin{array}{l}\text { Clustering } \\
\text { ('Traubenbildung') }\end{array}$ \\
\hline kein schwaches & $\begin{array}{l}\text { schwaches } \\
\text { Nominativpronomen }\end{array}$ \\
\hline Nominativpronomen & $\begin{array}{l}\text { NP-Status } \\
\text { Wortstatus oder Affix } \\
\text { Kopfstatus, also X }\end{array}$ \\
\hline syntaktische Affigierung & $\begin{array}{l}\text { lexikalische Affigierung } \\
\text { (Inkorporation) }\end{array}$ \\
\hline
\end{tabular}

Das negative Merkmal bei 'Traubenbildung' scheint von Belang insofern, als sich darin der Status eines freien lexikalischen Morphems mit eigener lexikalischer Semantik und syntaktischen Selektionsbeschränkungen verrät; Traubenbildung dagegen hat mit morphologischer Affigierung zu tun, die nach allgemeiner Einsicht gegenüber kategorialen Selektionen weniger empfindlich ist. Es ist weiter für eine reine VSO-Sprache erwartbar, daß sich nur ein Subjektpronomen in CL-Enklise zeigt; das Arabische hat mit der Mischung aus VSO- und SVO-Merkmalen Raum für ein freies DP-Subjekt im Nominativ, der in [Spez, AgrS] lizensiert ist. Schließlich ist die Enklitisierung im Arabischen insofern weiter fortgeschritten, als sich aus den enklitischen Positionen bereits durch Inkorporation Personalendungen am Verb ergeben haben. Damit zeigen sich in (22) Gemeinsamkeiten aus der gemeinsamen VSO-Geschichte der drei Sprachen ebenso wie Unterschiede aufgrund des modernen typologischen Wandels des Arabischen und Hebräischen.

\subsection{CL-Traubenbildung}

Zur CL-Traubenbildung im Deutschen und dazu, daß Trauben ('clusters') nicht disjungiert werden dürfen, vgl. (24). 
HOCHALEMANNISCH (MONTAFON)

(24) a *Gutsili hot-r göschtr-m ko:ft

b Gutsili hot-r-m göschtr ko:ft

Bonbons hat $=$ er $=$ ihm gestern gekauft

c

${ }^{*}$ Gutsili hot göschtr-r-m ko:ft

NEUGRIECHISCH (Drachman 1994, S. 4ff.)

(25) a (aftos) tu-to -dixni

(er) ihm-es-zeigt

b *(Ego) tu-sixna ta-exo dosi

(Ich) ihm oft ihnen habe gegeben

c *To-thelo na agoraso

es-will-(ich) SUBJUNCTIVE kaufe(-ich)

\subsection{Partielle Konjunktionskongruenz}

Nicht alle, nämlich nur die zweiten Personen im Singular und Plural, kongruieren mit der Verbpersonalendung und zwar regelhaft durch Kopierung der Verbflexion, in der ein Personsuffix steckt (so unter Wiederaufnahme älterer rein philologischer Beschreibungen bei Bayer 1994/85): (e)s, der alte Dual für den Plural $i h r, t(u)$ für den Singular. Wenn wir die Konjunktion in COMP nur als phonologischen Träger betrachten, den syntaktischen Ort aber unterhalb von COMP in AgrS bzw. AgrO annehmen (wie bereits in Abraham/Wiegel 1993; s. auch Abraham 1994: Kap. 11), erklärt sich der Zusammenfall von (i) klitischem Personalpronomen, (ii) Verbalsuffix in der syntaktischen Satzzweitstelle, in die ja das Verb rücken muB, sofern diese Stelle nicht durch eine Konjunktion besetzt ist, und (iii) der bestimmten, unverwechselbaren Stelle von CL (bei V oder bei COMP). Gleichzeitig spüren wir damit den janushaften Status von CL als Verbaffix und Personalpronomen auf, auf den ja in der traditionellen Indogermanistik schon immer hingewiesen wurde (Wackernagel 1892).

(26) a $\quad \ldots$ ob-t-s e:s kum-t-s

b ... wann-st (du) arbat-st

Dies sollte nicht zuletzt zweierlei zeigen: zum einen, daß die CLVerbaffixe (Verbinkorporationen und Restrukturierungen; vgl. DobrovieSorin 1994, S. 62) im Romanischen kein Einzelfall sind und daß sich solche Affigierungen eigens und gegen die allgemeine (greenbergsche) typologische Hypothese begründen (also spezifisch gegen 'SVO hat Proklise'); und zum andern, daß sich hier in statu nascendi vor unseren (freilich mundartlich gewieften) Augen ein Grammatikalisierungsproze $B$ 
anbahnt, der bisher nur in toten Sprachen mehr erschlossen als beobachtet wurde.

4. Gemeinsamkeiten zwischen dem Romanischen und Germanischen: Pronominalklitika (CL)

(27) CL \& schwache (atonische) Pronomina, nicht jedoch starke (tonische) Pronomina:

a nicht-fokussierbar (nicht nach $\mathrm{P}$; nicht kontrastiv betonbar) ( $\neq$ tonische Pronomina)

b nicht modifizierbar (= Vollpronomina auBer bei Relativsätzen)

c keine Konstituentenkoordination ( $\neq$ Vollpronomina)

d nicht in Satzspitzenposition (in Isolation?)

e haben den Status von N ('Kopfstatus'), nicht von NP (komplexen Konstituenten)

f morphonologisch reduziert (keine notwendige Bedingung)

g lassen sich nicht 'dislozieren' ('herausstellen')

(28) Eigenschaften (nur) von CL, im Unterschied zu stark-tonischen (nicht jedoch zu schwach-tonischen) Pronomina:

a morphologisch reduziert

b syntaktisch Köpfe (legen den Kategorienstatus des Komplexes fest)

c und nicht frei, d.h. kein Vorkommen in:

Positionen für koreferentielle NPs

in Isolation, innerhalb PPs, in Herausstellung, Spaltkonstruktionen

... Koordination, Modifikation, Kontrastfokus

d nicht notwendig [+animate] (Cardinaletti/Starke 1994)

(29)

\begin{tabular}{|c|c|c|}
\hline & XP & frei \\
\hline $\begin{array}{l}\text { klitisch } \\
\text { (CL) }\end{array}$ & - & - \\
\hline $\begin{array}{l}\text { starkes } \\
\text { Pron }\end{array}$ & + & \\
\hline
\end{tabular}


(29) b

\begin{tabular}{|lll|}
\hline & -frei & +frei \\
\hline $\mathrm{XP}$ & $?$ & stark \\
\hline $\mathrm{X}^{\circ}$ & $\mathrm{CL}$ & $\begin{array}{l}\text { kanonische } \\
\text { Köpfe }\end{array}$ \\
\hline
\end{tabular}

Die Stellung starker pronominaler Elemente im Deutschen ist identisch mit der der koreferenten Objekte - diese stehen ohne spezielle Kontextverknüpfung rechts innerhalb von VP. Es ist wieder zu betonen, daß dies besser an Dialekten zu zeigen ist, da nur dort echte CL vorkommen und somit die Stellungsunterschiede deutlich werden. Vgl. (30)-(33).

(30) Gebundene Prons in Spitzenstellung IN V-2

\begin{tabular}{|l|c|c|c|}
\hline & V-2-INITIAL & $\begin{array}{l}\text { FREI (z.B. IN } \\
\text { ISOLATION) }\end{array}$ & $\begin{array}{l}\text { NUR } \\
\text { MENSCHLICH }\end{array}$ \\
\hline $\begin{array}{l}e: r \text { 'er' } \\
\text { stark, tonisch }\end{array}$ & + & + & + \\
\hline $\begin{array}{l}\text { es 'es' } \\
\text { schwach, atonisch }\end{array}$ & + & - & - \\
\hline$a-s / z$ 'er - es' CL & - & - & - \\
\hline
\end{tabular}

\section{SÜDTIROLER DIALEKT}

[eckige Klammern für strukturelle VP-Grenzen]

(31) a $\quad \ldots \mathrm{daB}=\mathbf{a} / \mathbf{r}$ immer [INTELLIGENT isch]

$$
\mathrm{COMP}=\mathrm{CL}
$$

b ...?? daß e:r intelligent isch FOKUSSIERTES VOLLPRONOMEN

(32) a E:r isch immer [intelligent] PRONOMEN IN TOPIKPOSITION; FOKUSSIERT

b $\quad{ }^{*} \mathrm{a} / \mathrm{r}$ isch immer [intelligent] CL IN TOPIKPOSITION

(33) a Ich habe gestern [ ${ }^{*}$ sie/SIE getroffen] FOKUS AUF PRONOMEN IN VP

b Ich habe sie gestern [getroffen] SW. PRON AUSSERHALB/LINKS VON VP

c Ich habe gestern wirklich [SIE getroffen] 


\section{Thema-Rhema-Verteilung im (SOV-) Satz - topologische Felder}

\subsection{Integration der Diskurskategorien in die Satzstruktur}

Gehen wir von folgender, für eine Sprache wie das Deutsche weiter sicher unkontroverser diskursfunktionaler Struktur aus. Man beachte, da $B$ die Satzgliedzuordnungen zu den diskursfunktionalen Perioden nur sehr grob vorgenommen worden sind. Wohl sind bereits die Vorkommensbeschränkungen der CL angedeutet - mit der Unsicherheit in der Dimension 'Thema-Hintergrund' (?CL), die daher rührt, daB wir nicht wissen, wie sich diese diskursfunktionale Periode satzsyntaktisch im Detail strukturieren läßt.

\begin{tabular}{|l|l|l|l|l|}
\hline \multicolumn{5}{|c|}{ einfacher Satz } \\
\hline \multicolumn{3}{|c|}{ Thema/Topic } & \multicolumn{3}{c|}{ Rhema/Comment } \\
\hline Fokus & Hintergrund & & Fokus & Hintergrund \\
\hline ADVERB & FINITES V & & DO & (Nicht-) \\
& AUXILIAR & & PP & Finites V \\
& SUBORDINATOR & & NEG & \\
& ?CL & CL & ${ }^{*}$ CL & ${ }^{*}$ CL \\
\hline
\end{tabular}

Diese CL-Einreihung basiert auf zweierlei empirischen Vorgaben: einmal den Distributionseigenschaften, die wir bisher beobachtet haben (sowohl anhand der Literatur als auch hier in Beispielreihen vorgeführt); und zum andern auf der Einsicht, daB pronominale CL nur mit hohem diskursspezifischem, d.h. hochthematischem, referentiell vorgegebenem (in Uriagerekas Sinne 'spezifischem') Status verwendbar sind. ${ }^{11}$ Vgl, zum

11 Ich habe diese Einsicht nur in zwei Fällen in der linguistischen Literatur wiedergefunden: einmal explizit bei Givón (1976), das andere Mal implizit bei Uriagereka (1995, S. 90). Letzterer schreibt den Anlaß für den weiter in der Literatur unkontroversen 'Linksruck' von CL deren Spezifizitātsfunktion zu. Es bleibt aber völlig undiskutiert, ja es wird nicht einmal die Frage gestellt, warum Spezifizität nun außerhalb von VP angesiedelt ist. Nur zu vermuten ist, daB Uriagereka eigentlich an Spezifizität im diskursfunktionalen Sinne insofern denkt, als spezifische und definite NPs mit Satznormalakzent außerhalb von VP stehen, während nichtspezifische, indefinite NPs (Subjekte wie Objekte) ihren Basisplatz innerhalb von VP haben. Diese Opposition lāßt sich aber diskursfunktional verallgemeinern als logisch-semantische Interpretation von CL und sw. Pronomina innerhalb des kontextsensitiv strukturierten Satzes: also als strukturelle Periode für Satzthema im Unterschied zum Satzrhema. Siehe zu Begründungs- 
Themastatus der klitischen Pronomina bereits Givón (1976). Im besonderen sind CL - das haben die Distributionstests eindeutig erwiesen nicht in VP, offenbar der Satzperiode besonderer diskursfunktionaler Unspezifizität, d.h. von hochrhematischem Status, anzusiedeln. Was die genauere Position in der zweiten diskursfunktionalen Periode mit den Klassemen 'Thema' und 'Hintergrund' anlangt, so läßt sich jedenfalls sagen, daß es um den Rechtsrandbereich, also den Übergangsbereich zwischen der zweiten und dritten Spalte gehen muB. Die genaue Position ist ohne eine leistungsfähigere Syntax nicht zu erheben.

ansātzen und vielschichtiger Nachweisführung Abraham (1992; 1994, Kap. 14; Abraham 1995a, b). Immerhin kann auch Uriagereka nicht anders als im Zusammenhang mit einer bestimmten, CL motivierenden Funktionskategorie im Satz an eine Diskursfunktion the speaker's point of view zu denken. Er spricht in diesem Zusammenhang von einem syntaktischen Element „interfacing with pragmatic indexicality” (Uriagereka 1995, S. 93). 
(35) Satzfeldtypologische Einordnung von CL, schwachen und starken Pronomina:

CL und sw. Pron. in der Themaperiode, st. Pron. ebenso wie Voll-NPs in der Rhemaperiode. Winkelklammern zu lesen als "entweder $\langle A\rangle$ oder $<\mathrm{B}>$ (oder $<\mathrm{C}\rangle$ )" .

\begin{tabular}{|c|c|c|c|c|c|c|c|c|c|c|c|}
\hline \multirow{3}{*}{$\frac{2}{2}=$} & 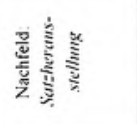 & 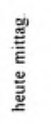 & & & & & 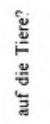 & 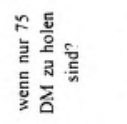 & 列 & & \\
\hline & 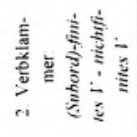 & 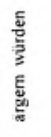 & 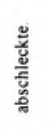 & 莺 & 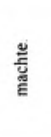 & 营 & 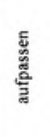 & & 竎 & & 跑 \\
\hline & : & 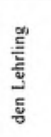 & 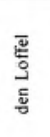 & & 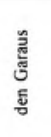 & & 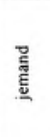 & & & 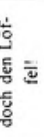 & \\
\hline \multirow{7}{*}{ 部 } & 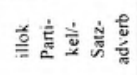 & हू & : & 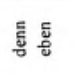 & & 产 & हू & 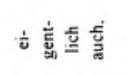 & 兵 & & 离 \\
\hline & 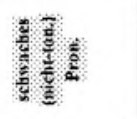 & s & s. & 88 & 淠 & a: & 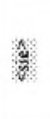 & $\frac{5}{2}$ & ) & & : \\
\hline & 8 & 8 & 3 & \& 1 & 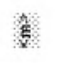 & & d) & y. & * & & \\
\hline & 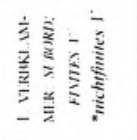 & 8 & 漚 & 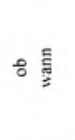 & tั & $\begin{array}{l}\text { 䓌 } \\
\underline{\underline{n}} \\
\underline{\underline{n}}\end{array}$ & $\underline{\underline{1}}$ & 壹 & 氖 & 5 & 퐆 \\
\hline & 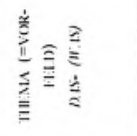 & n & $\frac{5}{\frac{5}{5}}$ & 憘 & 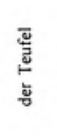 & 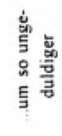 & & & & & \\
\hline & 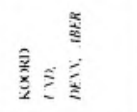 & & ஜே & & & & dِّ & $\stackrel{p}{5}$ & & & \\
\hline & $\underline{z}$ & & & & & & & & & & \\
\hline
\end{tabular}




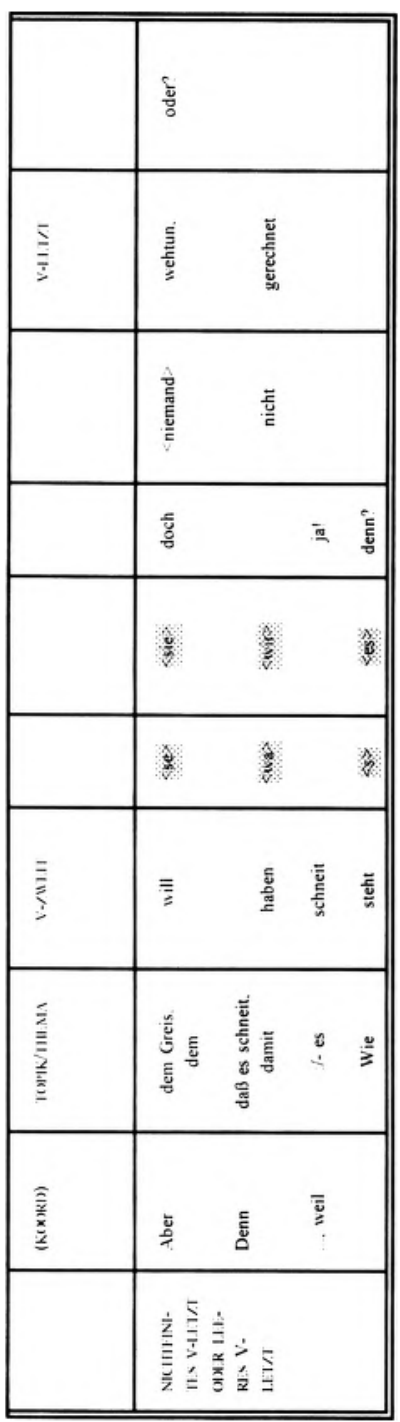




\section{Kasusunterschiede und lineare Nichtinvertierbarkeit}

Die Basisabfolge bei Objekten ist Dativ vor Akkusativ, bei schwachen Pronomina und CL jedoch Akkusativ vor Dativ. Die folgenden Abfolgebeschränkungen sind zu beobachten:

*Er hat ihm-DAT ihn-ACC überreicht \#(dem-DAT) Rotkäppchen den-ACC Korb

b *Sie will ihm-DAT es-ACC nicht sagen \#(dem-DAT) Rotkäppchen das-ACC Versteck

c ${ }^{*}$ Die Eltern wollten ihnen-DAT sie-ACC nicht lassen

(37) a ?'Er hat ihr-n (DAT-ACC) überreicht

b ${ }^{*}$ Er will ihm-n nicht zeigen

c Sie will ihm-s nicht sagen

d 'Er hat ihr ihn überreicht

e *Er will ihm ihn nicht zeigen

f *Sie will ihm es nicht sagen

Haegeman (1993) für das Westflämische und Vikner (1991) für die skandinavischen Sprachen zeigen, daß dieser Objektwechsel nicht auf das Deutsche beschränkt ist. Eine genauere Scheidung nach phonologischen Eigenschaften mag freilich ausweisen, daß hierbei auch nichtsyntaktische Bedingungen mitspielen - etwa daß Klitikkonsonanten mit schwacher Sonorität vor solchen mit höherer Sonorität stehen müssen (also /s/ vor $/ \mathrm{n} /, / \mathrm{n} /$ vor $/ \mathrm{m} /$ usw.; vgl. $(37 \mathrm{~b}, \mathrm{c}))$. Freilich liegt hiermit eine gemischte, syntaktisch-phonologische Bedingungstraube vor. Sollten sich die Beobachtungen oben nicht als vollständig erweisen (so die Behauptung von Lenerz 1992) und zu gemischten Distributionen führen, dann ist nur der SchluB zu ziehen, daB schwache Pronomina infolge von phonologischen oder idiomatischen Merkmalen flach konfiguriert sind, also keinen strukturellen Lizensierungsbedingungen unterworfen sind. Auch im Frz. zeigen sich solche reversiblen Abfolgen - doch vgl. auch (38c-e), aber eben gegen (38f):

(38) a Je le lui dirai

ich es ihm sage-FUT

b Je te le dirai

ich dir es sage-FUT

c Gib-s ihm!

d Gib ihm-s!

e Er hat-s-m/n-m gemacht

f Er hat-m-s $/{ }^{*} \mathbf{m}-\mathbf{n}$ gemacht 
Sollten jedoch die pronominalen syntaktischen Reversibilitätsverteilungen - eventuell neben phonologischen Nebenerscheinungen - allgemein haltbar sein, dann sind syntaktische Lösungen erforderlich. Diese Lösung ist komplex (Abraham/Wiegel 1993; Abraham 1994, S. 531ff.): sie geht davon aus, daß die Dativvalenz in einer Adjunktschale zu VP steht und $\mathrm{daB}$ somit der VP-interne Akkusativ über das Dativadjunkt nach links rückt, daß der Dativ als Linksadjunkt zur rhematischen VP andererseits stehen bleiben kann, da er eben nicht im Rhema-VP steht, das seinerseits nicht der diskursstrukturelle Ort für die fokusschwachen Thema-CL ist. Man beachte, daB die Datenbeobachtungen (nominale Dative vor Akkusativen, jedoch pronominal-klitische Akkusative vor Dativen, dies in weit über einzelne Sprachen hinausgehenden Beobachtungen, damit die Vorbehalte bei Lenerz 1992 entwertend) folgenden Schluß erzwingen: Dative stehen nach statistisch signifikanten Zählungen (Givón 1976, S. 160) in der Topikalisierungshierarchie deswegen höher, weil sie stärker referentiell-definit und durch die Eigenschaft [+menschlich] vertreten sind als Akkusative. Dies scheint mit ihrem prototypischen BENEFAKTStatus zusammenzuhängen und dies wiederum mit dem relativ stärkeren Anteil am Zustandekommen des Ereignisses der Satzprädikation. Nun ändert sich an diesen übergrammatischen Beziehungen natürlich nichts, wenn die Nomina durch Pronomina oder deren klitisch schwache Formen ersetzt sind. Trotzdem wechselt die Topikreihung so typisch und mit stark übereinzelsprachlicher Tendenz. Dies läßt nur die Folgerung zu, da $\beta$ Pronomina und CL nicht mehr wie freie Lexeme im Satz den topikalisierungshierarchischen Bedingungen unterliegen, sondern in grammatische Bindungsprozesse eingebunden, soz. grammatikalisiert sind: eingebunden eben in Inkorporationsprozesse mit kategorialer Trägerabhängigkeit, wie morphologische Köpfe der Art, wie sie hier beschrieben wurden. Auf den minimalistischen Erklärungsrahmen bezogen könnte dieser Wechsel freilich bedeuten, da $B$ der Akkusativ als struktureller Kasus zu einem funktionalen Knoten aufrücken muß, der Dativ als inhärenter Kasus dagegen VP-intern (oder VP-nahe) stehenbleibt. Ich diskutiere dieses Szenario hier nicht weiter (vgl. allgemein zur Kasuszuweisung im Rahmen der minimalistischen Syntax Abraham et al. (1996)).

\section{Enklise vs. Proklise, nichtdeklarative Konstruktions- typen und der Finitheitsparameter}

\subsection{Unterschiede}

Die allgemeine Stellungsthese zu CL in Abhängigkeit von den greenbergschen Abfolgetypen erfordert auf jeden Fall eine Präzisierung. Bereits auf ein paar wenige Sprachen beschränkt ergibt die CL-Verteilung nach 
bestimmten Konstruktionsbedingungen ein Bild, das aufs erste der allgemeinen typologisch begründeten These widerspricht. Dies betrifft die drei folgenden Konstruktionstypen: Imperative, Gerundien und Infinitive. Es zeigen sich folgende CL-Umgebungen bzw. Korrelationen (z.T. unter Rückgriff auf Daten bei Drachman 1994, Dobrovie-Sorin 1994 und Uriagereka 1995). Man vgl. (39).

\begin{tabular}{|l|l|l|l|}
\hline Proklise bei finitem & Infinitiv & Imperativ & Gerundium \\
Prädikat. & & & \\
\hline Französisch & $\mathrm{CL}+\mathrm{V}$ & $\mathrm{V}+\mathrm{CL}$ & $\mathrm{V}+\mathrm{CL}$ \\
Italienisch & $\mathrm{CL}+\mathrm{V}$ & $\mathrm{V}+\mathrm{CL}$ & $\mathrm{V}+\mathrm{CL}$ \\
Spanisch & $\mathrm{V}+\mathrm{CL}$ & $\mathrm{V}+\mathrm{CL}$ & $\mathrm{V}+\mathrm{Cl}$ \\
Neugriechisch & $\mathrm{CL}+\mathrm{V}$ & $\mathrm{V}+\mathrm{CL}$ & $\mathrm{V}+\mathrm{CL}$ \\
Portug. unabhgg. Satz & $\mathrm{V}+\mathrm{CL}$ & $\mathrm{V}+\mathrm{CL}$ & $\mathrm{V}+\mathrm{CL}$ \\
Portug. abhgg. Satz & $\mathrm{CL}+\mathrm{V}$ & - & - \\
\hline Deutsch & $\mathrm{CL}+\mathrm{V}$ & $\mathrm{V}+\mathrm{CL}$ & $\mathrm{CL}+\mathrm{V}$ \\
\hline Enklise bei finitem Prädikat & Infinitiv & Imperativ & Gerundium \\
\hline
\end{tabular}

Ganz offensichtlich erfordern diese Daten eine feinkörnigere Analyse als das weniger feinkörnige greenbergsche Raster mittels $\mathrm{S}, \mathrm{V}$ und $\mathrm{O}$. Außerdem liefert diese tiefergehende syntaktische Analyse nicht nur den Schlüssel dazu, daß sich die allgemeinere These (unter dem gröberen greenbergschen Raster) halten läßt, sondern sie erhärtet auch den diskursfunktionalen Erklärhintergrund.

Die herausfallenden und erklärungsbedürftigen Abfolgen sind unten typographisch hervorgehoben: im spanischen Infinitiv wie in (40) im Vergleich zum übrigen romanischen, etwa im Französischen wie in (41), im unabhängigen Satz des Portugiesischen sowie im gesamten Deutschen als einzigem Vertreter des SOV/SVO-Typs (d.h. eines Typus mit relativ strenger V-letzt-Abfolge im abhängigen Satz und gleichzeitig ganz strenger V-zweit-Abfolge im unabhängigen Satz).

(40) a Lo oimos es hörten(-wir) „Wir hörten es"

b Para oirlo zu hören=es

(41) a Tu le feras du es tun-FUT „Du wirst es tun”

b Elle va le faire sie geht es tun „Sie wird es tun" modernes Spanisch

modernes Frz. 
(42) a

$$
\begin{aligned}
& \text { a Levá-lo-ei } \\
& \text { hebe(ich)=es=FUTURAUX } \\
& \text { b Mostra-no-los-á } \\
& \text { zeigen=uns=sie-FUTURAUX }
\end{aligned}
$$

europ. Portugiesisch

„Ich werde es heben”

Zum Portugiesischen habe ich hier nichts weiter zu sagen (dazu vgl. Rıvero 1988; Madeira 1992), auBer daß man sich durch scheinbar subjektlose Deklarative wie in $(42 a, b)$ oben nicht darüber hınwegtäuschen lassen darf, daB hier tatsächlich echte SVO-Strukturen vorliegen. Die pronominalen thematischen Subjekte sind ja flexivisch erkennbar. Freilich sind keıne Proklitika beobachtbar, sind keine topıkalisierten phonologisch starken Morpheme vorhanden, die als Träger der CL fungieren könnten. Dies ist eine der syntaktisch strukturellen Einschränkungen, denen die allgemeine These zur typologischen Verteilung zwischen Proklitika und Enklitika unterliegt. Die grammatische Alternative ist somit Inkorporation in die Verbflexion.

Im Deutschen wäre, gerechnet nur nach der SOV-Typologie, gerade ein Stellungsspiegelbild zum Romanischen zu erwarten gewesen - das sich mit den Abfolgen im Infinitıv und im Imperatıv jedoch nicht einstellt, Im Gerundıum dagegen ist die Abfolge 'erwartungsgemäß' splegelbildlıch zum Romanischen Was ist der tiefere Sinn dessen?

\section{Imperative}

Imperatıve verzeichnen $\mathrm{Im}$ allgemeinen die folgenden Eigenschaften, sofern nicht bloB Sprechaktfunktion und nicht ein eigenes morphologisches Flexionsparadıgma den Imperatıvcharakter bestımmt (1) sie erscheinen nur in unabhängıgen Sätzen, (ii) sie haben demgemäß nıemals eıne einlettende Konjunktion (in COMP) oder ein anderes einlettendes Satzglied wie ein Adverb (in Sonderheit die Negationspartikel - im Deutschen wird gerade mit Negation gerne der Inıfinitıv verwendet. nicht schlagen!, was erklärungsbedürftig ist); (iii) die Imperativformen stehen am absoluten Satzbeginn - vorabgehende NPs sind offenbar als Themata pendentia lınksherausgestellt. Den überzeugendsten Nachwess für die Top-Stellung des Imperatıvs liefern SOV-Sprachen wie das Deutsche, das Niederländische und das Westfriesische - erwarten wir doch nach der Zweitstellung des imperativischen Prädikats CL-Enklise, die sich auch ausnahmslos einstellt

In Strukturen mit V-Top-Stellung wie den Imperativen muB auch im Romanischen das Verb in COMP stehen - Im Gegensatz zu allen anderen Strukturen, wo es in IP steht ${ }^{13} \mathrm{Vgl}$ (43) (Dobrovie-Sorın 1994, S 59f).

${ }^{13}$ Im Deutschen dagegen steht das finite $V$ außer in Nebensātzen, wo COMP 
(43) a FRZ. regarde-le

b RUM. da-i-l mit der Struktur [Comp regarde $\left.i_{i}=l_{k}\right]\left[\begin{array}{lll}I P & t_{k} & t_{i}\end{array}\right]$

gib- ihm-ihn

c trimite-mi-o

schick- mir-sie

Diese Imperativstrukturen werden wie gesagt deshalb eigens vermerkt, weil sie ja der zentralen, aber eben durch die zweite These strukturell spezifisch eingeschränkten Verallgemeinerung $z u$ widersprechen scheinen: bei strengem SVO stehen CL proklitisch. Wie wir sehen, bezieht sich dieser allgemeine Schluß auf SVO mit IP-Stellung von V (also der Verbstellung im deklarativen Satz), nicht jedoch auf Strukturen mit V in CP (dem Konjunktionsknoten), also einem Knoten über IP, dem Satzflexionsknoten (im Imperativ muß das Verb in die Satzspitzen-, die TOPPosition, um dort sowohl der Satzoperatoren- wie auch einer Fokusposition zu genügen). Der diskursfunktionale Grund für die Linksverrückung ist gleichwohl erfüllt: die pronominalen CL sind in (43a-c) ja aus VP heraus nach links gerückt und stehen adjungiert an das finite Verb in COMP. Man sieht dies nur mit der oberflächlichen traditionellen Grammatikbrille nicht, weil die Klitika in gleicher Weise rechts von V stehen! Ein wichtiges Weiteres läßt sich aus (43a-c) oben ableiten: wenn die CL einzelne strukturelle Köpfe - etwa in $\mathrm{I}^{\circ}$ und in $\mathrm{C}^{\circ}$ - wären, dann könnte das Verb bei seinem Linksrücken in eine TOP-Position auch zwischen den beiden CL stehen bleiben. Das ist aber völlig ausgeschlossen. Damit liegt der Schluß nahe, daß die beiden CL ins Verb inkorporiert sind. Dieser Schluß wird fürs Deutsche - und soviel ich sehe ausschließlich fürs Deutsche - auch gestützt durch die Kongruenzbeobachtungen für Konjunktionen (Bayer 1984/85; Abraham 1994, S. 521), also genau an der Kategorie, wo sich auch die CL adjungieren und wohin das finite Verb rücken muß, sofern diese Stelle nicht (wie im konjunktionseingeleiteten Nebensatz) durch eine Konjunktion besetzt ist. Solche scheinbaren Enklitika wie in (43a-c) haben also einen anderen Strukturaufbau als die normal beobachteten Proklitika. Vgl. (44) (nach Dobrovie-Sorin 1994, S. 61).

(44) a Ausgangsposition für SVO wie das Rumänische:

COMP ... [IP CL [IP V-I [vP tV ...]]]

durch die Konjunktion besetzt ist, vermutlich immer in COMP. Das sieht man nicht ohne weiteres. 
b proklitische Inkorporationsrestrukturierung ('restructuring incorporation'):

COMP ... [IP [I, CL V-I [vP tv ...]]]

c enklitische morphologische CL-Prädikateinschmelzung ('merger'):

[сомP V-I $=\mathrm{CL}$ [IP $\mathrm{t}_{\mathrm{CL}}\left[\mathrm{IP} \mathrm{t}_{\mathrm{V}-\mathrm{I}}\right]$ ]]

Proklitika sind trotz der unverrückbaren Adjazenz zum finiten $V$ freie Morpheme, in ihrer Selektionsposition dem Artikel vergleichbar; vgl. (44b). Enklitika dagegen haben worteingebundenen Charakter, sind somit unfrei, stärker verbkongruenzmorphologisch in ihrem Charakter. ${ }^{14}$ Soweit zu Imperativen, die sich quer durch alle Sprachen darin gleichen, daß das imperativfinite Verb als Satzoperator an die absolute fokussierende Satzspitze rücken muß und dabei CL auf jeden Fall in einer Themaposition landen läBt.

Gelegentliche Proklitika beim Imperativ sind entweder als herausgestellt (Thema pendens) oder als topikalisiert, also noch innerhalb des Satzgefüges, zu betrachten. Man vergleiche die beiden neugriech. mundartlichen Imperative (von Chios: $(45 \mathrm{a}, \mathrm{b})$ ) in derselben Bedeutung "Halte deinen Mund!" (nach Drachman 1994, S. 13). Die bedeutungsgleichen Entsprechungen des Standardgriechischen zeigen bloß die kanonische Proklise (vgl. $(45 c, d))$ :

$\begin{array}{lll}\text { a } & \begin{array}{l}\text { ta-loya-su ta-thu } \\ \text { die Worte-deine sie=halte } \\ \text { thun-ta ta-loya-su }\end{array} & \text { Dialekt von Chios; Proklise(!) } \\ \text { b } & \text { Dialekt von Chios; Enklise } \\ \text { halte=sie die Worte-deine } & \\ \text { c } & \begin{array}{l}\text { krata-ta ta-loya-su } \\ \text { d }\end{array} & \text { *ta-loya-su ta-krata-k }\end{array}$

Die Negationspartikel steht nie links vom (d.h. strukturell höher als das) Verb (Drachman 1994, S. 13). Da Neg andererseits strukturell höher als der funktionale Kongruenzknoten liegt, muß das imperativische Prädikat in COMP stehen.

\subsection{Gerundien}

Gerundien verhalten sich nach (39) in allen hier beobachteten Sprachen gerade umgekehrt, als man es nach der allgemeinen typologischen Stellungsthese (V-Randsprachen Enklise, V-Mittelsprachen Proklise) erwar-

14 Einen, wie mir scheint, ganz ähnlichen, nur in anderem Jargon ausgedrückten Unterschied trifft auch Uriagereka (1995), indem er im Sinne der Minimalismustheorie $\mathrm{z}$ wischen starken und schwachen $\mathrm{CL}$-attrahierenden Kategorien bzw. dem parametrischen Fehlen soicher Kategorien unterscheidet. 
ten würde: der romanische SVO-Typus zeigt Enklise, der deutsche SOVTypus dagegen Proklise. Zum Deutschen vgl. man:

(46) a zum's/es ihm mit einem Blumenstrauß Überreichen

b zum'n/ihn den Safe sauber aufbrechen Lassen

c zum ihm <'s/?es> mit einem BlumenstrauB <*'s/*es> Überreichen

d zum den Safe <*'n/*ihn> sauber <*'n/*ihn> aufbrechen Lassen

CL ist nur mit dem präpositionalen Träger $z u m$ verträglich. Wenn die nominale Verbform mit ihren Valenzen im VP-Status ist und die Adverbiale mit einem Blumenstrauß bzw. sauber linke VP-Grenzmarken darstellen, dann steht die Präposition zum im Satzflexionsknoten IP oder in COMP oder irgendwo dazwischen. Wenn wir davon ausgehen, daB Gerundien zumindest Aspekt, wenn nicht sogar Tempus (aber eben nicht Person und Numerus) ausdrücken, also in den IP-Knoten vorrücken, ohne AgrS zu erreichen, dann steht $C L$ in der deutschen Gerundialkonstruktion auf jeden Fall in der thematischen Satzperiode.

Ganz anders jedoch haben wir den anderen Gerundialtypus zu analysieren. Ich orientiere mich am Neugriechischen (Beispiele und Analyse nach Drachman 1994, S. 12f.).

\section{NEUGRIECHISCH (Drachman 1994, S. 12):}

a to grama-su, ematha oti i-Maria, diavanzondas-to, yelase dein Brief, ich hörte, daß die Maria lesend ihn

b ematha oti o-Petros fevgondas pire ta klidya mazi-tu ich erfuhr daB Peter weggehend nahm die Schlüssel mit sich

c * to grama-su, ematha oti i-Maria, diavanzondas-to yelase (kein Komma vor yelase!)

d Sie hat den ganzen Kaffee zum Trinken ausgeschüttet.

e Sie hat das Kind das ganze Flascherl, zum $\mathrm{t}_{\mathrm{i}}$ Austrinken gezwungen.

f Sie hat $\left\langle{ }^{*}{ }^{\prime} s_{\mathrm{i}}\right\rangle^{15}$ das Kind $\left\langle{ }^{*} \mathrm{~s}_{\mathrm{i}}\right\rangle\left\langle\right.$ das ganze Flascherl $\left.\mathrm{l}_{\mathrm{i}}\right\rangle$ zum $\left\langle\right.$ 's $\mathbf{s}_{\mathrm{i}}>$ Austrinken gezwungen.

Drachman nennt auch das adverbiale Konverb ohne Rektion in (b) 'Gerundium'. Wir beschränken uns hier auf die objektregierende Form in $(a, c)$ sowie das Deutsche in $(d-f) .(a, c)$ sowie (f) zeigen Objekt-CL. Im Deutschen weisen die Stellungsbeschränkungen nach (f) die PP-interne

15 Winkelklammern kodieren alternative Verwendung, also 'entweder - oder - oder ...'. 
Position des Objekts als Basisposition aus. Im hochnormalisierten Hochdeutschen gilt keine der Alternativen (zwischen $<>$ ) - um überhaupt zu Daten zu gelangen, muß man die gewachsene Umgangssprache bemühen. Zum Griechischen schließen wir, nur z.T. Drachman folgend, folgendes. Daß nur die Version mit Parenthese grammatisch ist, bestätigt nur die Position, daß nichtfinite Konstruktionen ein (unsichtbares) PROSubjekt mit Nullkasus (also nicht mit Nominativ!) haben müssen. Da in (c) das Subjekt i-Maria realisiert ist, ist gegen diese Bedingung verstoßen. Dies erklärt einerseits den Grammatikalisierungsunterschied zwischen (a) und (c). Andererseits zeigt die grammatische Parenthese deutlich, daß keine finite Konstruktion, im besonderen keine Satzflexionskategorie (Agr-Knoten) vorliegt. Damit ist diese Gerundialstruktur in (a) genauso ein adverbielles Konverb wie die in (b). Solchen tempus-, modus- und numeruslosen Strukturen lassen sich jedoch keine Diskursfunktionen wie Thema vs. Rhema zuordnen. Dann aber braucht CL das Rhemagebiet, nämlich die VP, auch nicht zu verlassen. Es bleibt folglich in seiner Grundposition unter Rechtsrektion, nämlich kanonisch rechts von V. Man prüfe anhand von (47), daß im Gerundium auch fürs Deutsche die unter Linksrektion gültige Position gilt. Da alle romanischen Sprachen für nicht V-topikalisierende Strukturen vom SVO-Typus (wo das Verb in IP bzw. dessen Subselektion AgrP steht, nicht in CP wie im Deutschen) sind, gilt durchgehend und völlig erwartbar ' $V+C L$ '. Das deutsche präpositionale Gerundium mit ausschließlichem P-CL zeigt letztlich, daß kongruenzlose CP-Strukturen existieren - CL hängt sich an eine Kategorie an, die sonst COMP als CL-Träger vorbehalten ist. Und wenn man Gerundien nominalen Kategorialstatus zuspricht, dann zeigt ebendies, daß es nominale CP-Strukturen gibt - was freilich keinen logischen Semantiker (perseverierenden 'Lambdakonvertiten') überrascht.

Es bleibt darauf hinzuweisen, daß die beiden Enklisen für den Imperativ (ebenso wie den Interrogativ) und das Gerundium trotz gleicher Oberflächenposition auf völlig unterschiedliche Mechanismen zurückgehen; die einen, Imperativprädikate, haben nominativische pro-Subjekte und stehen an der Satzspitze; die anderen dagegen, Gerundien, haben kasuslose PRO-Subjekte (wie in Kontrollstrukturen unter Verben wie versprechen/raten/befehlen + Infinitiv) und stehen keineswegs an der Satzspitze - sie sind ja nichtfinit nach Person, Numerus und eventuell Tempus (sicher nicht jedoch nach Aspekt). Und letztlich ist zu beachten, da $B$ die CL-Enklise quer durch alle Sprachen, in Sonderheit die der sonst proklitischen romanischen CL-Realisierer, auf eine Verbvorrückung über alle möglichen Landeplätze nach links zurückgeht - weiter als sonst in den romanischen Deklarativsätzen nötig ist. Somit ergibt sich eine the- 
matisch diskurskodierte Landestelle schon vor dem imperativisch-finiten V.

\subsection{Infinitive}

Mit 7.2-7.3 ist auch klar, inwiefern das oberflächlich gemeinsame Merkmal der Nichtfinitheit bei Gerundien und Infinitiven für den diametralen Unterschied (Enklise beim roman. Gerundium, Proklise beim Infinitiv mit Ausnahme des Spanischen) keinen erklärenden Status besitzt (d.h. über die allgemeine typologische These hinausgehen muB). Infinitive haben kein PRO, das ja wie bei der Kontrollkonstruktion regiert sein muB (Er $r_{\mathrm{i}}$ versprach $\mathrm{PRO}_{\mathrm{i}} z u$ kommen). Ohne Subjekt und ohne Kongruenz besteht kein AnlaB für V, überhaupt aus seiner durch die Thetarollen bestimmten VP-Position in den Kongruenzknoten ins Mittelfeld zu rücken; es bleibt rechts von CL in V-Letztposition, wie es die Rektionsläufigkeit deutscher Verbaktanten vorschreibt (Abraham 1994, Kap. 1). Ob am Beginn des Infinitivkomplexes stehende CL-Formen in die syntaktische Argumentation miteinbezogen werden sollten, steht dahin: es wäre dann ein Subjekt-pro vorauszusetzen, das alleine thetasemantisch begründet ist und für die Lizensierung von CL herzuhalten hätte.

$$
\begin{aligned}
\text { (48) a } & -\mathrm{s}-\mathrm{m} / \mathrm{n} \text { gern gebm } \\
& =\text { es=ihm gerne gegeben } \\
\text { b } & -\mathrm{s} \text { den gern gebm } \\
& =e s \text { dem gerne gegeben } \\
\text { c } & { }^{*} \text { den-s gebm } \\
\text { d } & \text { eam des } /{ }^{*} \text { es gebm }
\end{aligned}
$$$$
\text { bairisch-österreichisch }
$$

den "dem" bzw. des "das/es" ist die schwache Pronominalform, die in einer Vielzahl deutscher Dialekte das schwächste Glied im Paradigma, $e s$, dann vertritt, wenn dieses nicht als syntaktisches CL auftritt. ${ }^{16}$ Die Alternative wäre, den Status derartiger Formen ausschließlich phonologisch, also als Schnellsprechformen festzulegen.

Syntaktische Subjektlosigkeit ist der Grund, weshalb die gleichermaßen rektionsrechtsläufigen romanischen SVO-Sprachen Gerundial- und Infinitiv-CL grundlegend asymmetrisch konstruieren. Wieso fält aber das Spanische mit $V+C L$ aus dem allgemeinen Infinitivbild der Romania heraus? Wir können im Augenblick nur so viel sagen, daB CL in span.

16 es kommt in der schwachen und der starken syntaktischen Verteilung nicht oft vor und wird in solchen Umgebungen im allgemeinen durch $d$-Formen ersetzt. Von schwäbischen Kollegen (U. Lutz/Tübingen) habe ich mir sagen lassen, daß man seinen Dialekt gerade aufgrund dieser Ausnahme erkennt. Zu dialektalen Varianten des Deutschen vgl. Abraham/Wiegel 1993. 
para oirlo verbinkorporiert ist und als Affix nur in Kopfstellung, also rechts vom Verbstamm stehen kann. Uriagereka (1988) bringt dies mit der Existenz eines parametrisch agierenden Funktionsknotens in Verbindung bzw. der Attraktionskraft dieser Funktion derart, daß ein freies CL-Morphem oder ein Verbaffix entsteht (s. auch Uriagereka 1995, S. 92ff.).

\subsection{SchluBfolgerung}

Der zentrale Schlüssel für die auseinanderlaufenden Positionen der pronominalen Klitika (CL) ist die Finitheit des Prädikats nach den $\mathrm{Be}-$ stimmungsstücken Tempus, Subjektperson und Subjektnumerus bzw. die spezifische syntaktisch-semantische Funktion des CL-regierenden Prädikats. Beim Imperativ (ebenso wie beim Interrogativ, gleichgültig ob in der Wortfrage oder Satzentscheidungsfrage) muß das Prädikat in die Satzoperatorposition in CP rücken, um den spezifischen grammatikalisierten Imperativsprechakt zu lizensieren. Das imperativisch-finite V steht also strukturell höher als im einfachen Deklarativsatz, für den der Greenbergtypus SOV bzw. SVO ausgelegt ist (dies, so wissen wir alle, in Ermanglung einer detaillierteren und leistungsfähigeren Syntaxstruktur). In einer solchen Top-Position des V braucht aber auch bei SVO (dem Typus, dem alle der romanischen Sprachen in (39) angehören) CL nicht höher als bis eben außerhalb von VP (genauer in die strukturelle Dimension von IP oder AgrP) nach links zu verrücken. Somit bleibt die allgemeine, auf Deklarativstrukturen ausgelegte These unbeschadet: SVO zeigt Proklise. Im speziellen Imperativfall müßte man greenbergisch von VSO und damit von Enklise ausgehen - was ja mit der Hauptthese im Einklang ist.

8. Die germanisch-skandinavischen Sprachen vom SVOTypus mit Enklise - CL-Ausscherer?

Das Schwedische zeigt CL-Nachbindung, obwohl es vom SVO-Typus ist. Widerspricht dies unserer Generalisierung?

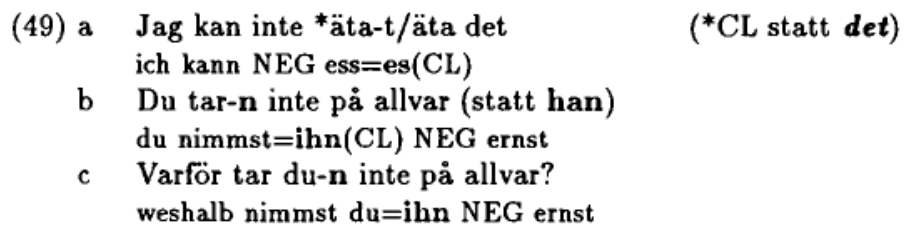


Das Schwedische stellt trotz seines unkontroversen SVO-Typus die schwachen und klitischen Pronomina postverbal (aber vor die Satznegation). Vgl. auch (50) fürs Norwegische.

(50) NORWEGISCH (Christensen-Koch 1985):
a Hun $/{ }^{*} \mathrm{a}$ har bodd her
${ }^{*} \mathrm{CL}$ in Satzspitzenstellung sie $/{ }^{*} \mathrm{CL}$ hat gewohnt hier
b Vi vet at ikke hun $/{ }^{*}$ a har bodd her
${ }^{*}$ CL nach NEG wir wissen daB NEG sie hat gewohnt hier
c Vi vet at a ikke har bodd her CL im Mittelfeld, vor NEG

Ohne hier näher darauf eingehen zu können gehe ich davon aus, daB die Position des starken Satznegators eine strukturelle Grenze setzt. Im Keltischen (VSO) steht ein solches NEG, ket (als Partikellexem, nicht als proklitischer Satzaffirmator präverbal wie im Bretonischen), postverbal vor Objekten, Adverbien, Objektnebensätzen oder Prädikatspartizipien, jedenfalls aber vor dem Kongruenzkomplex, IP (vgl. neuerdings Schafer 1995, S. 161 et passim). Auch in den romanischen SVO-Sprachen steht die starke Neg-Partikel oft direkt vor den Objekten: man denke an pas direkt hinter dem finiten Verb im Frz., ohne daB sich ein anderes Satzglied zwischen dieses NEG und das finite Verb drängen kann. Starkes NEG setzt zum finiten Prädikatsteil einerseits und zur Kette von Objekten andererseits eine enge Beziehung, allerdings in Abhängigkeit von der typischen Rektions- und Satzskopusrichtung: nach rechts bei SVO-Sprachen, nach links bei SOV. Man denke an das Deutsche, wo die NEG-Partikel nicht durch nichts von der V-letzt-Position getrennt werden kann und sich auf alle definiten Objekte links von NEG bezieht.

$$
\begin{aligned}
& \text { a weil <*nicht }>\text { er }<* \text { nicht }>\text { [vP [den Nachbarn }<\text { nicht }> \\
& \text { b-S-Skopus] ausstehen kann] } \\
& \text { b because he <*not }>\text { often does [s-Skopus }-><\text { not }><* \text { often }> \\
& \text { [vP participate at parties]] }
\end{aligned}
$$

Das Wesentliche ist, daß zwischen der unabhängigen und der abhängigen Satzstruktur eine auffallende Asymmetrie in dem Sinne besteht, daß sich im abhängigen Satz ein 'Mittelfeld' (nach Diderichsen (1962) ein eigenes 'Nexusfeld') öffnet, in das schwache Adverbien rücken können (Platzack 1988). In dieser strukturellen Periode landen CL in der erstmöglichen Stelle auf ihrer Suche nach einer Themaposition - durchaus im Sinne der speziellen CL-These in (ix), zusammengefaßt nochmals in (xv) unten. Eine solche Asymmetrie besteht in den anderen SVO-Sprachen, den romanischen Sprachen und dem Englischen, nicht; entsprechend muß CL 
über das finite Prädikat weiter nach links rücken. Man vgl. nochmals die norweg. Beispiele in (50).

\section{Schluß: Erklärung für die CL-Verschiebung vor dem Hintergrund von Diskursfunktion und Satztypologie}

(xi) Für strenge SVO-Sprachen, also Sprachen ohne stellungsfreies Subjekt ist die THEMATISCHSTE Position die des Subjekts. Jedes andere Satzglied links vom Subjekt steht außerhalb des einfachen Satzes (etwa als Thema pendens); andere Satzglieder als Subjekte sind vor dem finiten V oder AUX im allgemeinen nicht gestattet. Thematisches Material, sofern nicht selbst $S$, steht $z$ wischen $S$ und $V$. Es muB links von $V$ stehen, denn $\mathrm{V}+\mathrm{O}$ ist ja RHEMATISCH. Diese Situation führt zu PROKLISIS. Sie gilt für die romanischen SVO-Sprachen. Die kanonische phonologische Verschleifung mit dem finiten Verbträger spricht dafür, von einem Vorschritt zu Präfigierung zu sprechen. Ohne Zweifel ist die Proklise im Bretonischen - das ja eine keltische Sprache und ursprünglich vom Typus VSO ist - durch arealen Einfluß des Französischen (SVO) zu erklären.

(xii) Die Merkmalskombination [+stellungsfreies $\mathrm{S},+\mathrm{SVO}$ ] wie im Slawischen (sofern diese Sprachen die CL als Erscheinung überhaupt aufweisen; das Russische etwa hat keine pronominalen CL, wohl aber Nullsubjekte) erlaubt jedes Satzglied in der Topikposition. Auch in solchen Sprachen steht CL links von V, allerdings nicht in präfigaler Bindung (also als Morphem freier). Auch solche Sprachen zeigen jedoch PROKLISIS.

(xiii) Der SOV/SVO-Typus der kontinentalen westgermanischen Sprachen (Deutsch, Niederländisch, Westfriesisch, Jiddisch) sowie der ebenfalls V-randständige VSO-Typus des Walisischen hat eindeutig ENKLISE, unabhängig davon ob S stellungsfrei ist oder nicht. Er zeigt ENKLISE, weil die Position zwischen V und $\mathrm{S}$ die am weitesten linke bzw. vorderste ist, bis zu welcher ein THEMATISCHES Element aufsteigen kann in dem Bestreben, seine Diskursfunktion zu signalisieren. Beim Walisischen ist erwägbar, daB sein VSO aus einem früheren indoeuropäischen SOV (V[SO] aus [SO]V) stammt. Weiter sieht es so aus, als verhielte sich der VOS-Typus (nach dem Befund der australischen Eingeborenensprachen; vgl. Schachter 1976) wie VSO. Der exotische Typus ist jedenfalls SVO!

(xiv) Auch das Semitische (kairoter Umgangsarabisch, modernes Hebräisch) zeigt ENKLISE der pronominalen CL, jedoch mit bestimmten Unterschieden zum Walisischen. Diese Unterschiede sind auf den gemischten 
typologischen Status des modernen Arabischen und Hebräischen (SVO mit VSO-Optionen) zurückzuführen.

(xi)-(xiv) bestätigen die eingangs aufgestellte rein empirische allgemeine CL-Hypothese, daB die V-randständigen Sprachtypen ENKLISE, die Vmittelständigen dagegen PROKLISE aufweisen. Dies ist jedoch insofern zu korrigieren bzw. zu präzisieren, als es nichtdeklarative Satzbilder gibt, in denen auch die V-mittelständigen Sprachtypen CL-Enklise zeigen. Wir haben veranschaulicht, daB dies dann vorliegt, wenn die finite Prädikatkomponente in die Topikposition vorrückt. Andererseits gibt es den Vmittelständigen Typus wie die kontinentalskandinavischen Sprachen mit CL-Enklise (statt der für diesen Typus vorausgesagten Proklise). Dieser Umstand zwingt dazu, die allgemeine These zugunsten der spezielleren aufzugeben.

(xv) SPEZIELLERE CL-THESE: Pronominale CL sind hochthematisch. Sie stehen demgemäB in jener thematischen Position, die den geringsten Abstand zu den koreferenten nominalen Entsprechungen aufweist. Im Szenario eines Verschiebungsmechanismus der CL aus der VP heraus nach links heißt dies, daB CL in der erstmöglichen thematischen Position stehen bleibt. Wir haben diese diskursstrukturelle Satzperiode, in der das thematische pronominale CL zu stehen kommt, FP genannt und es weiterer empirischer Arbeit vorbehalten, diese vorerst diskursfunktionale Kategorie FP jeder Sprache extra auf die universalgrammatisch relevanten strukturellen Satzperioden abzubilden.

In den kontinentalskandinavischen V-mittelständigen Sprachen ist der Ort dieser diskursfunktionalen CL rechts vom finiten Prädikat. Diese FPPeriode unterscheidet sich distributionell so von der der romanischen Vmittelständigen Sprachen, daß die in der Linksverrückung früheste thematische Bedingung bereits vor dem mittelständigen V, also in der Enklise, erfüllt ist. FP in den skandinavischen V-mittelständigen Sprachen liegt rechts vom finiten Satzprädikat, in den ebenfalls V-mittelständigen romanischen Sprachen dagegen links davon.

(xvi) Mit der Ausbildung von CL scheint ein gewisser Ausprägungsgrad der Kongruenzfunktion (nach Person und Numerus beim Verb) bei den CL-Trägern einherzugehen. Daraus folgt, daB AGR ausgeprägten, paradigmatisch hochidentifizierten Status hat. Das Englische hat kein solches hochentwickeltes Kongruenzparadigma, folglich kein pronominales CL. Auch wenn (zusätzlich) kasusidentifizierende Strukturpositionen für CL in Anspruch genommen werden (etwa bei Cardinaletti 1994, Abraham/Wiegel 1993, Abraham 1995), wird verständlich, daß das sowohl 
kongruenz- wie kasusfremde moderne Englische keine CL-Positionen bereitstellt.

Da im Altenglischen das Kongruenz- und Kasusparadigma noch ähnlich wie im Deutschen ausgebildet war, sollten syntaktische CL (und nicht nur phonologische wie in we're, they'll) vorkommen können. Eine der Schwierigkeiten bei toten Sprachen stellt die schreibsprachliche Überlieferung dar. Das moderne Griechische besitzt CL, wie wir dargestellt haben, das Altgriechische hingegen nicht, soweit wir dies beurteilen können - d.h. das Altgriechische verfügt nur über 'schwache Pronomina' (nach der bei Cardinaletti/Starke 1994 entwickelten Nomenklatur). Das Lateinische scheint bloB einen Personalpronomentypus zu besitzen, nämlich 'stark'. Sicheres verschließt sich uns bei alten Sprachen jedoch.

(xvii) Es ist nur natürlich, davon auszugehen, daß Null-CL-Sprachen ('pro-drop'-Sprachen) das Vorstadium der CL-Erscheinung kennen. Diese Annahme, so plausibel sie ist, wird einem aber durch verschiedene Sprachen schwer gemacht: etwa durch das Russische, das sehr wohl Nullprosubjekte kennt, uns aber in der Schriftsprache keinen CLAnhaltspunkt dafür zur Verfügung stellt. Dasselbe gilt für das Lateinische. Und: inwiefern erlaubt das Verbflexionsparadigma weniger eindeutige Verbkongruenzidentifikationen als das Italienische? Oder: wie läßt sich angesichts jedes Fehlens von verbaler Objektflexion die pro-dropErscheinung von Objekten in italienischen Dialekten erklären? Zwingen uns solche Einsichten dazu, den CL-Zwischenschritt auf dem Weg zu pronominalen Nullsubjekten aufzugeben? Oder fehlt in dieser Kalkulation ein Aspekt, den wir bisher völlig unterschlagen haben? Liegen hier einfach rein unsystematische pragmatische Auslöser zugrunde, die sich dann einfach konventionell - d.h. ohne tieferen typologischen Grund fixiert haben? ${ }^{17}$

17 Eine Erwägung in dieser Richtung ist folgendes wert. Man geht allgemein davon aus, daß pro-drop-Sprachen wie das Italienische, Rumānische, Portugiesische und Spanische, etwa im Unterschied zum Französischen hinreichend reich unterschiedene Verbkongruenzparadigmen aufweisen, um auf die Nennung des diskursfunktional schwachen Subjektpronomens verzichten zu können. Vgl. die Übersicht in (i).

(i)

Französ. Deutsch Italien. Neugriech.

starke Pronomina schwache Pronomina CL pro-drop

$\begin{array}{llll}+ & + & + & - \\ - & + & + & + \\ + & +(-) & + & + \\ - & - & + & +\end{array}$


(xviii) Worin in den einzelnen CL-Sprachen auf jeden Fall Gemeinsamkeiten bestehen, sind die folgenden Stellungskriterien (zu (a)-(c) vgl. Dimitrova-Vulchanova 1993, S. 23):

(a) sie lassen als CL-Träger nur einen Vertreter einer spezifischen grammatischen Kategorie zu;

(b) sie ordnen CL einer bestimmten linearen Position zu, bleiben aber inbezug auf die kategoriale Zugehörigkeit des CL-Trägers neutral;

(c) sie ordnen CL in eine größere CL-Traube ein;

(d) der CL-Position in der Satzstruktur, die ja die erste (vom linken VP-Rand aus gezählte) erreichbare thematische Position ist, kommt unterschiedlicher kategorialer Status zu und zwar eine zwischen der TOP-Position und eben dem linken VP-Rand. ${ }^{18}$

Diese Annahme machen solche Sprachen noch plausibler, die sich aufgrund von Objektkongruenz in der Verbmorphologie diskursfunktional schwache Nullobjekte leisten: etwa das Ungarische. Freilich scheint eine solche verbale Objektflexion gar nicht nötig zu sein, wenn wir solchen Erscheinungen wie in italien. Dialekten Glauben schenken. Nun ist das Deutsche aber auf jeden Fall unter den Sprachen, die sich aufgrund ihres Verbkongruenzparadigmas Nullsubjekte leisten könnten: vgl. bin - bist - ist - sind - seid - sind bzw. $-e-(e) s t-(e) t-e n-(e) t-e n$. Das erlaubt eine fast $100 \%$ ige Identifikation nach Person und Numerus. Das Französische identifiziert sogar noch vollstāndiger. Trotzdem fehlen Nullrealisierungen. Einen Schlüssel scheinen die Umgangssprache und Dialekte zu liefern. So kann man im Deutschen in Fragen und Ausrufen durchaus sagen: Bist (du) blöd!? Seid's (ihr/es) ganz gescheit?! Daß't/Wann'st nur aufpaßt!', in Notizen wie Bin um $5 \mathrm{zu}$ hause/habe schon gegessen, Sind bald zurück sowie in Erzählanfängen wie in Ist einmal ein kleines Männchen gewesen, Hat einmal ..... Natürlich gibt es auch hier Einschränkungen (so spielen diese Beispiele nie das gesamte Personparadigma durch); auch sehe ich von den mundartlichen Besonderheiten der Beispiele ab, ohne welche die Identifikation schlechter ginge. Es geht darum, daß die Subjektidentifikation eben auch ohne Subjektpronomen eindeutig wäre, sollte sich das Deutsche konventionell zur Nullrealisierung verstehen. Aber die Sprechergemeinschaft des Deutschen läßt sich diese Doppelidentifikation, 'diesen Luxus' eben nicht nehmen. Es ist durchaus zu erwägen, daß sich mit diesem Konservativismus - den ja z.B. eine so verwandte Sprachgemeinschaft wie die Niederländer nicht mit uns teilen - auch eine Typologie der Sprechergemeinschaften und der Sprachkultur erstellen ließe. Zu einem ersten Versuch in dieser Richtung vgl. Abraham (1995b).

Dimitrova-Vulchanova (1993) bestimmt diesen Ort fürs Slawische als kategorial offenen Satzknoten, der über der Negationskonstituente (die im Slawischen immer vorne steht) und der Moduskonstituente rangiert, die ihrerseits über dem Auxiliarkomplex und VP sitzt und zu dem sie für CL Rechtsadjunktion beobachtet. Uriagereka (1995) bestimmt diesen Ort rein 
Zwischen diesen vier Kriterien besteht keineswegs komplementäre Verteilung; vielmehr lassen sich verschiedene Kombinationen denken: etwa zwischen (a) (wie im Deutschen) und (b) (Wackernagelgeneralisierung). Das Deutsche beachtet ohne jeden Zweifel sowohl (a) wie auch (b). Ohne Zweifel wandeln sich Sprachen auch derart, daß sie vom einen Typus zum andern übergehen oder indem sie zwei Regelkriterien zulassen, die allerdings unterschiedlichen regional bestimmten Systemen entstammen. So beobachtet Dimitrova-Vulchanova $(1993$, S. 23) für das Makedonische Zugehörigkeit zu (c) (CL-Traubenbildung) wie auch (b), ersteres offenbar als Folge der analytischen Entwicklungen innerhalb des Balkansprachbundes (etwa bei Bulgarisch und Rumänisch). (b) im besonderen erlaubt die Verallgemeinerung, da $B$ die strukturelle TOP-Position im Satz von jedem Satzglied eingenommen werden kann, solange sie nur entsprechende Textanknüpfungsfunktionen verkörpern kann. Dies ist ja, wie wir am Englischen leicht ablesen können, nicht in jedem Sprachtypus gegeben (im Englischen etwa kann nur das grammatische Subjekt in dieser TOP-Position stehen; eigene fokussierte Topikalisierungen müssen über Spaltkonstruktionen realisiert werden). Die Wackernagelposition, die vor allem für die alten idg. Sprachstadien beobachtet wurde (Wackernagel 1892), gilt mit Sicherheit für eine große Zahl moderner Sprachen nicht, selbst für das in vieler Hinsicht konservative Deutsch nicht (vgl. Taylor (1993) zum Altgriechischen, das schon aus der Wackernagelverallgemeinerung ausschert). Es wäre sinnvoll, der Frage nachzugehen, ob diese Wackernagelposition als tendenziell optimale CL-Position an bestimmte satzstrukturelle, typologische Eigenschaften geknüpft ist. Es ließe sich bei einer solchen Forschungsstrategie z.B. die Idee verfolgen, daB SOVTypus mit V-Zweit vorliegen muB. Man denke bei diesem Ausgangspunkt an die alten Stadien der romanischen Sprachen wie das Altfranzösische, -italienische, -spanische sowie Altenglisch mit der SOV-Struktur, das im Unterschied zum modernen Französischen CL enklitisch an seinen phonologischen Träger band (Adams 1987, Fontana 1993). DimitrovaVulchanova (1993, S. 23) beobachtet, daB das Makedonische dabei ist, (b) (= Wackernagelbedingung, d.h. rein lineare Festlegung ohne kategoriale Eindeutigkeit) zugunsten des areal beeinflußten (c) aufzugeben.

Allgemein hat sich wieder gezeigt, daß diskursfunktionale Kategorien wie Thema, Rhema, Vorder- und Hintergrund nicht nur direkte Entsprechungen in der syntaktischen Struktur haben, sondern daß sich ihr jeweiliger syntaktisch-struktureller Ort auf der Grundlage einer hinreichend stark differenzierenden und empirisch-distributionellen Eigen-

kategorial als FP, lăßt aber nicht erkennen, worin eine allgemeine kontextuelle Identifikation dieser FP bestehen könnte. 
schaften Rechnung tragenden Unterscheidung festlegen läßt. Dies wurde hier vornehmlich fürs Deutsche vorgetragen; aber es besteht begründeter AnlaB, die diskurstechnische Struktur in (34) je nach den eigenen Distributionseigenschaften der einzelnen Sprachen ebenso satzsyntaktisch zu bestimmen.

Dies beschließt meine Ausführungen. ${ }^{19}$ Es sollte deutlich geworden sein, daB diese typologische Exerzitie - sofern ihr irgendein empirischer Wert zukommt - gezeigt hat, daB man typologisch ohne detaillierte Distributionskriterien einer Verallgemeinerung des CL-Ortes gar nicht nachhorchen kann, daB mit anderen Worten typologische Erörterungen auf der Basis der greenbergschen Unterscheidungen alleine zu keinem sinnvollen Ergebnis führen.

\section{Literatur}

Abraham, W. (1988a): Middle constructions in German. In: Groninger Arbeiten zur germanistischen Linguistik 28, S. 25-62.

Abraham, W. (1988b): Vorüberlegungen zur Syntax der Modalpartikeln. In: Linguistische Berichte 118, S. 443-465.

Abraham, W. (1993): Null subjects in the history of German: from IP to CP. In: Lingua 89/2-3, S. 117-142.

Abraham (1992a): The syntax of Thema and Rhema. In: Gilbers, D./ Looyenga, S. (eds.): Language and cognition II. Groningen Yearbook of Linguistics and Cognitive Rescarch. Groningen. S. 1-18.

Abraham, W./Wiegel, A. (1993): Reduktionsformen und Kasussynkretismus bei deutschen und niederländischen Pronomina. In: Abraham, W./Bayer, J. (Hg.): Dialektsyntax. Opladen. S. 12-49.

Abraham, W. (1994): Deutsche Syntax im Sprachenvergleich. Grundlegung einer typologischen Syntax des Deutschen. Tübingen. (Studien zur deutschen Grammatik 41).

Abraham, W. (1995a): Middle constructions in West Germanic. In: Abraham, W./Givón, T./Thompson, S. (Hg.): Discourse grammar and typology. Amsterdam. S. 1-43.

19 Was hinter weiteren Idiosynkrasien wie CL-X-CL-Dopplungen oder CL-XNP-Kopien diskurskausal steht, bleibt im Moment hier ohne Erklärungsversuch (dazu z.B. Dobrovie-Sorin 1994 fürs Rumänische; Shlonsky 1995 fürs Westflämische). Es gilt auf jeden Fall, Argumentpositionen von Nichtargumentpositionen zu unterscheiden. Wahrscheinlich entzieht sich ein Erklärversuch auch dem hier angepeilten diskursfunktionalen Hintergrund, so daB solche Fragen hier gar nicht in erster Linie zu verfolgen sind. Man würde nur eben gerne einen tieferen Grund vermuten wollen, als der bloße verallgemeinerte Beschreibungsproze $B$ auf der Grundlage einer rein formal begründeten Landeplatzproliferation herzugeben vermag. 
Abraham, W. (1995b): German cold and male - Dutch warm and female. Considerations of a typology of national speaking communities. In: Shannon,Th. F./Snapper, J. (Hg.): Biannual Berkeley Studies in Dutch literature and linguistics. Berkeley, California. S. 171-210.

Abraham, W./Einstein, S./Trainusson, H./Zwart, C.J.W. (in Vorb.) (Hg.): Minimal Ideas. Amsterdam.

Adams, M. (1987): From Old French to the theory of pro-drop. In: Natural Language and Linguistic Theory 5,1, S. 1-33.

Bayer, J. (1984/85): COMP in Bavarian syntax. In: The Linguistic Review 3, S. 209-274.

Belletti, A. (1994): Case checking and clitic placement. Three issues on (Italian/Romance) clitics. In: Geneva Generative Papers 1993, vol. 1/2, S. 101-118.

Cardinaletti, A. (1994): On the internal structure of pronominal DPs. In: The Linguistic Review 11/3-4, S. 195-220.

Cardinaletti, A./Starke, M. (1994): The typology of pronouns. A view from Germanic. Paper Harvard.

Christensen-Koch, Ch. (1985): Subject clitics and A'-bound traces. In: Nordic Journal of Linguistics 8, S. 12-30.

Diderichsen, P. (1962): Elementaer Dansk grammatik. Kopenhagen.

Dimitrova-Vulchanova, M. (1993): Clitics in Slavic. In: Frondheim Working Papers in Linguistics 18, S. 1-50.

Dobrovie-Sorin, C. (1994): The syntax of Romanian. Comparative studies in Romance. Berlin/New York. (Studies in Generative Grammar 40).

Drachman, G. (1994): Some properties of clitics (with special reference to Modern Greek). FAS/Berlin-Vortrag 1994. Paper Univ. Salzburg.

Evans, D.S. (1976): A grammar of Middle Welsh. Dublin.

Ethyrsson, Th. (1994): Functional categories, cliticization, and verb movement in the early Germanic languages. Paper for the Ninth Comparative Syntax Workshop, Harvard University. January 5-6, 1994.

Fanselow, G. (1991): Minimale Syntax. Groningen. (Groninger Arbeiten der germanistischen Linguistik 32).

Farkas, D./Zec, D. (1995): Agreement and pronominal reference. In: G. Cinque/Guisti, G. (Hg.): Advances in Roumanian linguistics. Amsterdam. S. 83-102. (Linguistics Today 10).

Fassi-Fehri, A. (1993): Issues in the structure of Arabic clauses and words. Dordrecht.

Fontana, J.M. (1993): Phrase structure and the syntax of clitics in the history of Spanish. Dissertation Univ. Pennsylvania, Pittsburg.

Givón, T. (1976): Topic, pronoun, and grammatical agreement. In: Li, Ch. (Hg.): Subject and topic. New York, S. 151-188.

Grosu, A. (1994): Three studies in locality and case. London/New York. (Theoretical Linguistics). 
Haegeman, L. (1993): Some speculations on argument shift, clitics, and crossing in West-Flemish. In: Abraham, W./Bayer, J. (Hg.): Dialektsyntax. Opladen. S. 131-160.

Hendrick, R. (1988): Anaphora in Celtic and Universal Grammar. Dordrecht.

Holmberg, A. (1991): The distribution of Scandinavian weak pronouns. In: Riemsdijk, H.v./Rizzi, L. (Hg.): Clitics and their hosts. Univ. of Tilburg.

Kayne, R. (1975): French syntax. Dordrecht.

Lewis, H./Pedersen, H. (1937): A concise comparative Celtic grammar. Gōttingen.

Lenerz, J. (1992): Zur Syntax der Pronomina im Deutschen. Lund. (Sprache und Pragmatik. Arbeitsberichte 29).

Madeira, A. (1992): On clitic placement in European Portuguese. In: UCLWP 4, S. 95-122.

Platzack, Chr. (1988): The emergence of a word-order difference in Scandinavian subordinate clauses. In: Fakete, D./Laubitz, Z. (Hg.): McGill Working Papers in Linguistics: Special issue on Comparative Germanic Syntax, S. 215-238.

Progovac, L. (1993): Clitics in Serbo-Croatian: COMP as the second position. Paper Wayne State University, read at LSA Meeting at Boston (in Sept. 1994).

Rivero, M.-L. (1988): The structure of IP and V-movement in the languages of the Balkans. Paper Univ. Ottawa.

Roberts, I./Shlonsky, U. (1994): Pronominal enclisis in VSO languages. Paper Bangor/Maryland/Geneva. (GLOW presentation 1994, Vienna).

Schachter, Paul (1976): The subject in Philippine Languages: topic, actor, actor-topic, or none of the above. In: $\mathrm{Li}, \mathrm{Ch}$. (Hg.): Subject and topic. New York. S. 439-518.

Schafer, R. (1995): Negation and verb-second in breton. In: Natural Language and Linguistic Theory 13, S. 135-172.

Shlonsky, U. (1994): Semitic clitics. In: Geneva Generative Papers 2/1, S. 1-11. (LSA talk 1993).

Shlonsky, U. (1995): Agreement in COMP. In: The Linguistic Review 11/3-4, S. 351-376.

Taylor, A. (1993): A prosodic account of clitic positions in Ancient Greek. Paper Univ. Pennsylvania.

Ternes, E. (1970): Grammaire structural du Breton de l'Ile de Groix. Heidelberg.

Thurneysen, R. (1966): A grammar of Old Irish. Dublin.

Uriagereka, J. (1988): On government. Dissertation University of Connecticut, Storrs.

Uriagereka, J. (1995): Aspects of the syntax of clitic placement in Western Romance. In: Linguistic Inquiry 26, 1, S. 79-124.

Vikner, St. (1991): Verb movement and the licensing of NP-positions in the Germanic languages. Dissertation Univ. Genf. 
Wackernagel, J. (1892): Über ein Gesetz der indogermanischen Wortstellung. In: Indogermanische Forschungen 1, S.333-436. 